\title{
Intermolecular Chirality Transfer from Silicon to Carbon: Interrogation of the Two-Silicon Cycle for Pd-Catalyzed Hydrosilylation by Stereoisotopochemical Cross-over \\ Sebastian Rendler ${ }^{\dagger}$, Martin Oestreich ${ }^{\dagger \star}$, Craig P. Butts ${ }^{\ddagger}$, and Guy C. Lloyd-Jones ${ }^{\ddagger *}$ \\ Organisch-Chemisches Institut, Westfälische Wilhelms-Universität Münster, Corrensstrasse 40, D-48149 Münster, Germany; Organic \& Biological Chemistry Section, School of Chemistry, University of Bristol, Cantock's Close, Bristol BS8 1TS, United Kingdom \\ E-mail: martin.oestreich@uni-muenster.de,guy.lloyd-jones@bristol.ac.uk
}

Supporting Information II

Copies of NMR spectra, MS spectra and HPLC traces 


(SiS) $-2 a$
rac-2a
$\mathrm{C}_{13} \mathrm{H}_{20} \mathrm{Si}$
$204.38 \mathrm{gi} / \mathrm{mol}$

rac-1-tert-Butyl-1-(1,2,3,4-tetrahydro)-1-silanaphthalene (rac-2a) and

rac-1-tert-Butyl-1-(1,2,3,4-tetrahydro)-1-silanaphthalene [(SiS)-2a]

ReOeM124-504,Rendler,SR191A,CDC13=7.26, $500 \mathrm{MHz}$

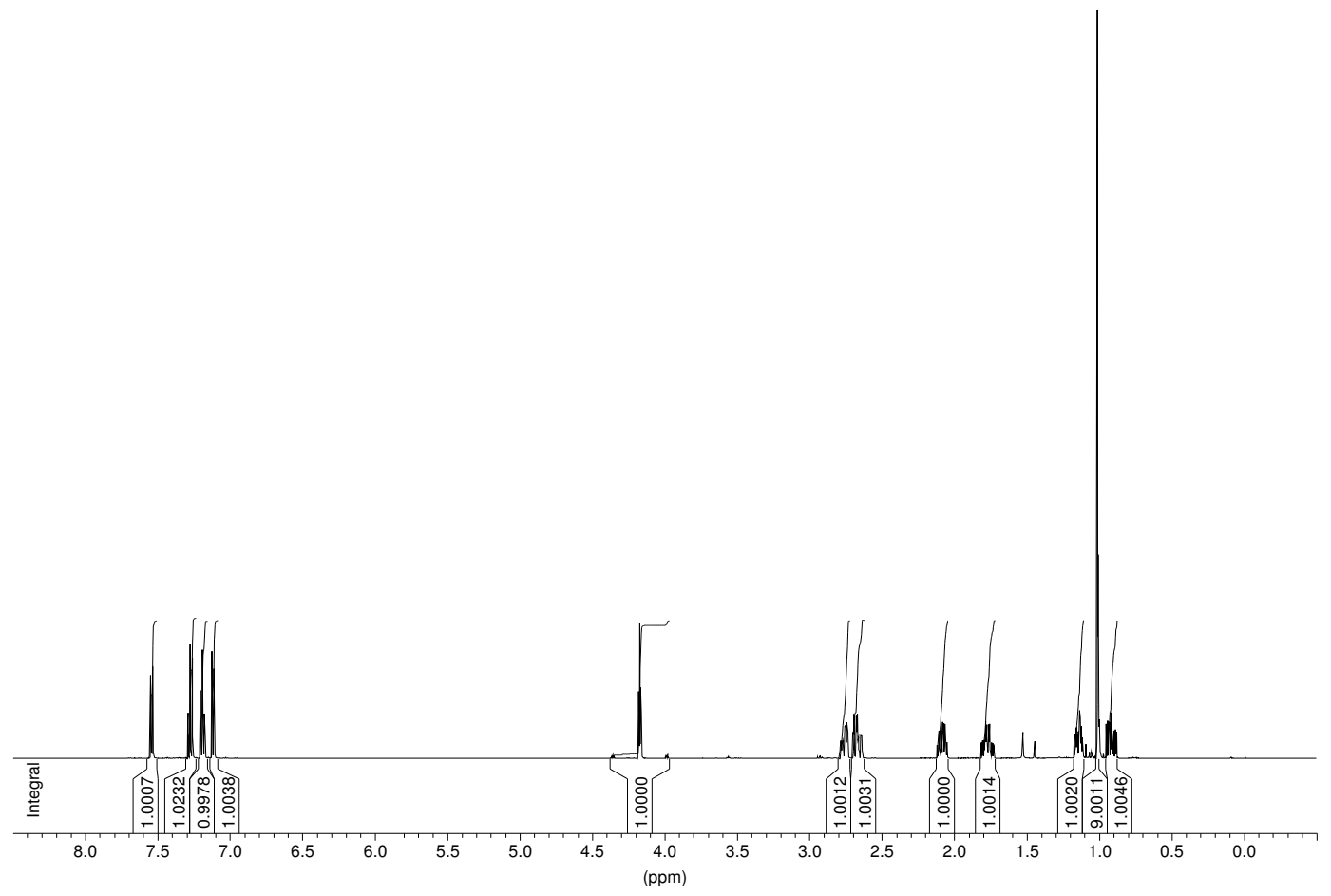


ReOeMI24-505, Rendler,SR191A,CDCl3=77.1000, $125 \mathrm{MHz}$
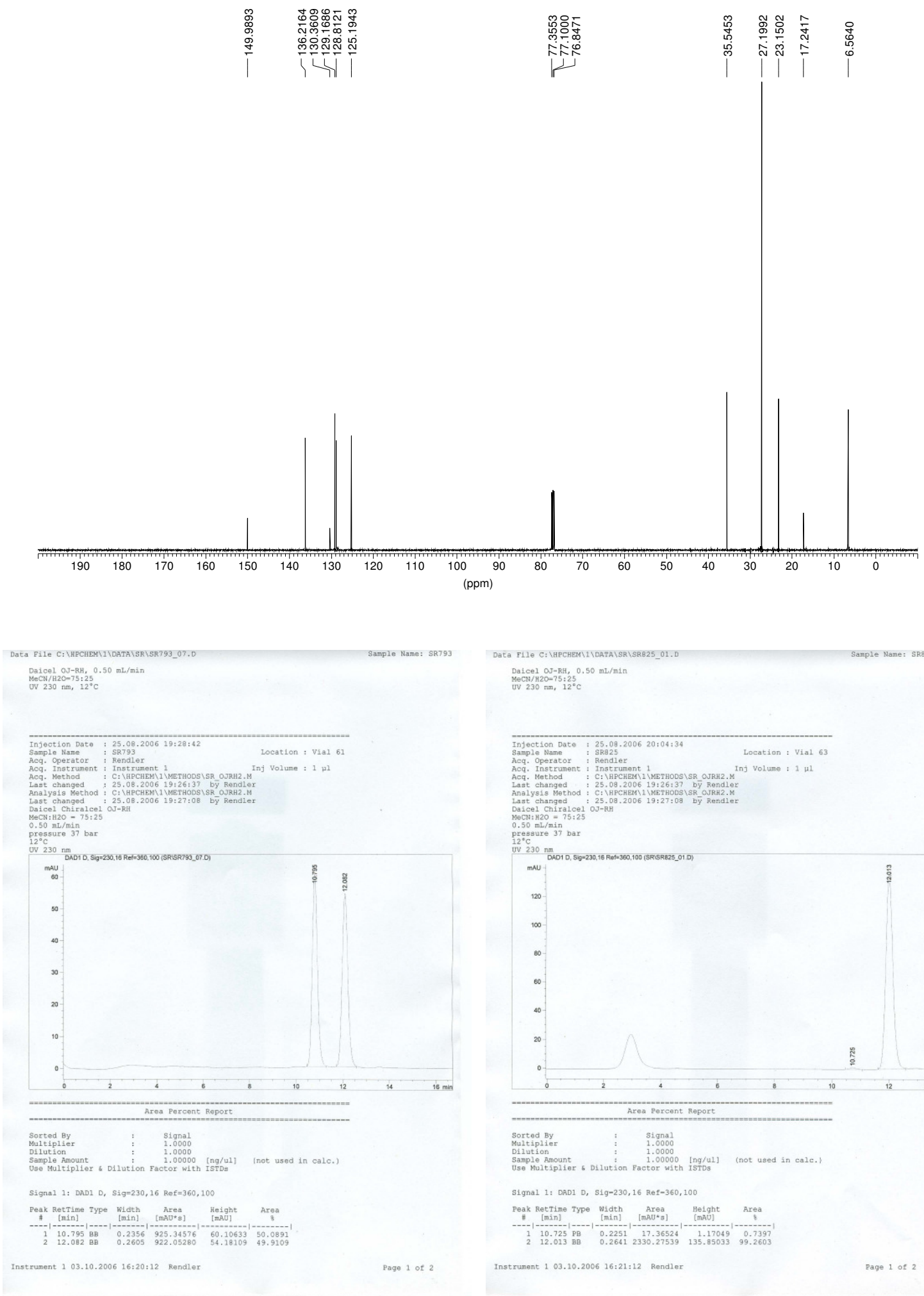

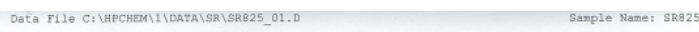

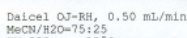

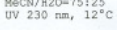

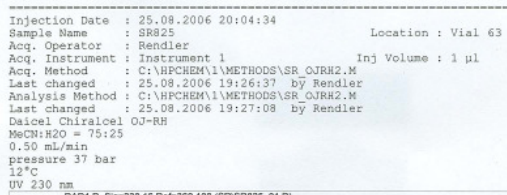

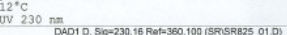

maU

120

100

$\infty$

${ }^{60}$

${ }^{40}$

${ }^{20}$
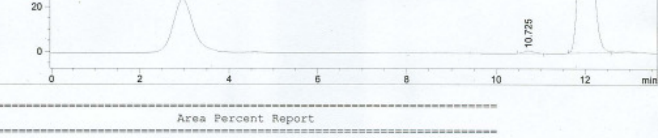

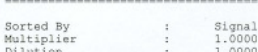

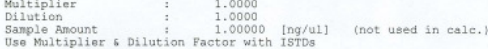

S1gnal 1: DADI D, S1g-230,16 Ref-360,100

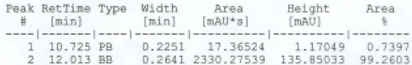

Instrument 1 03.10.2006 16:21:12 Rendle

Page 1 of 2 


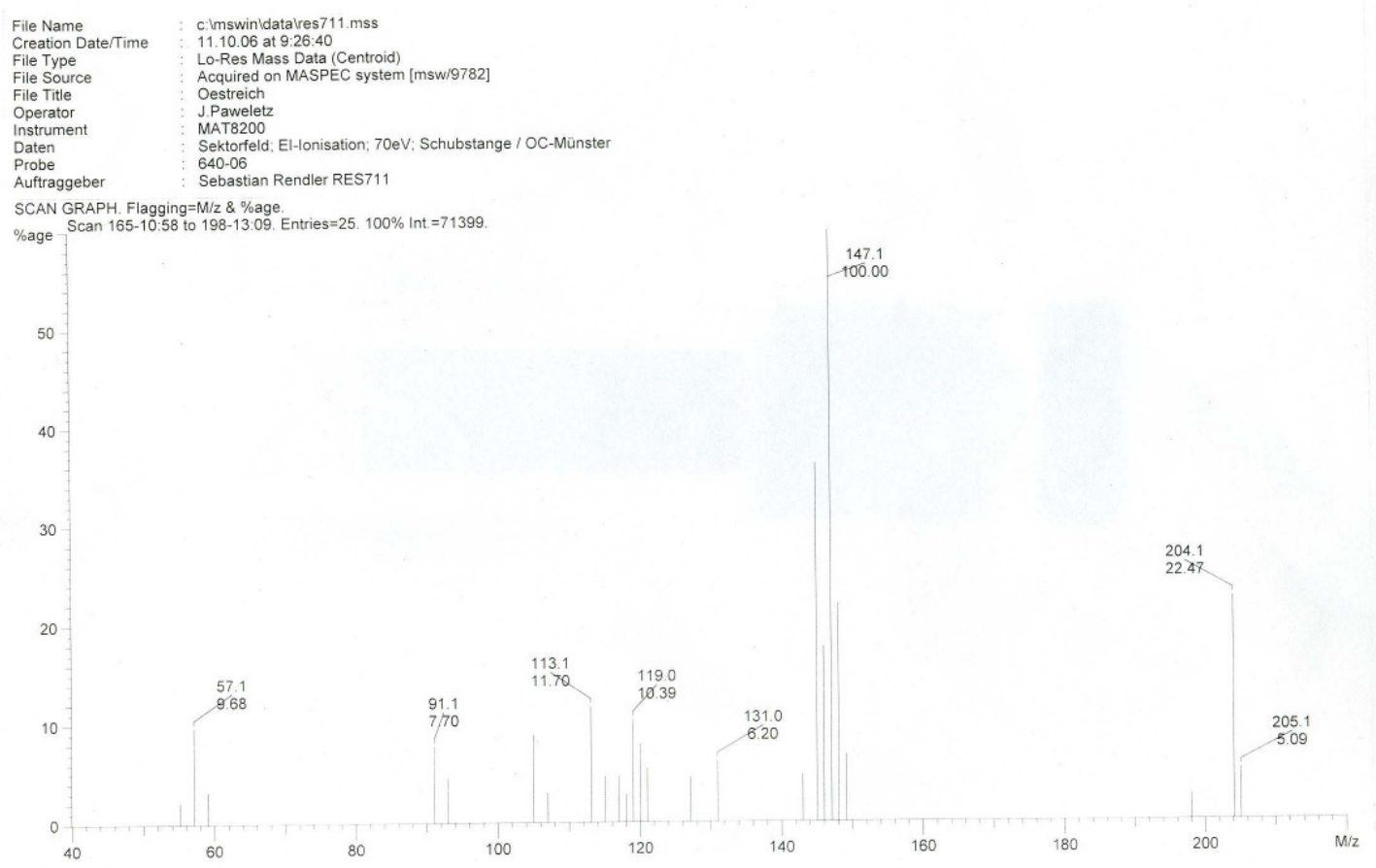




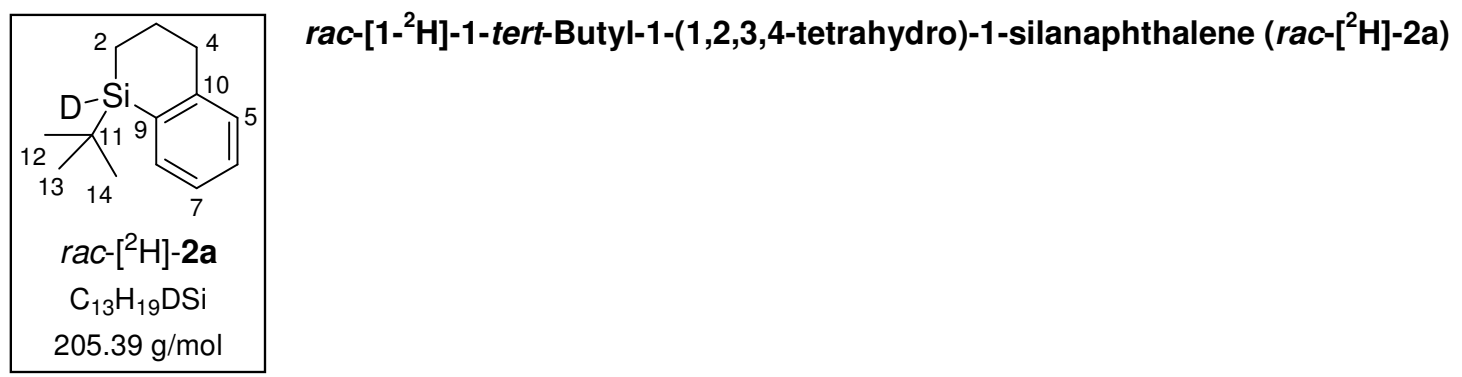

REOEAU26-501,RENDLER,SR-31IA,CDCL3=7,2600ロ

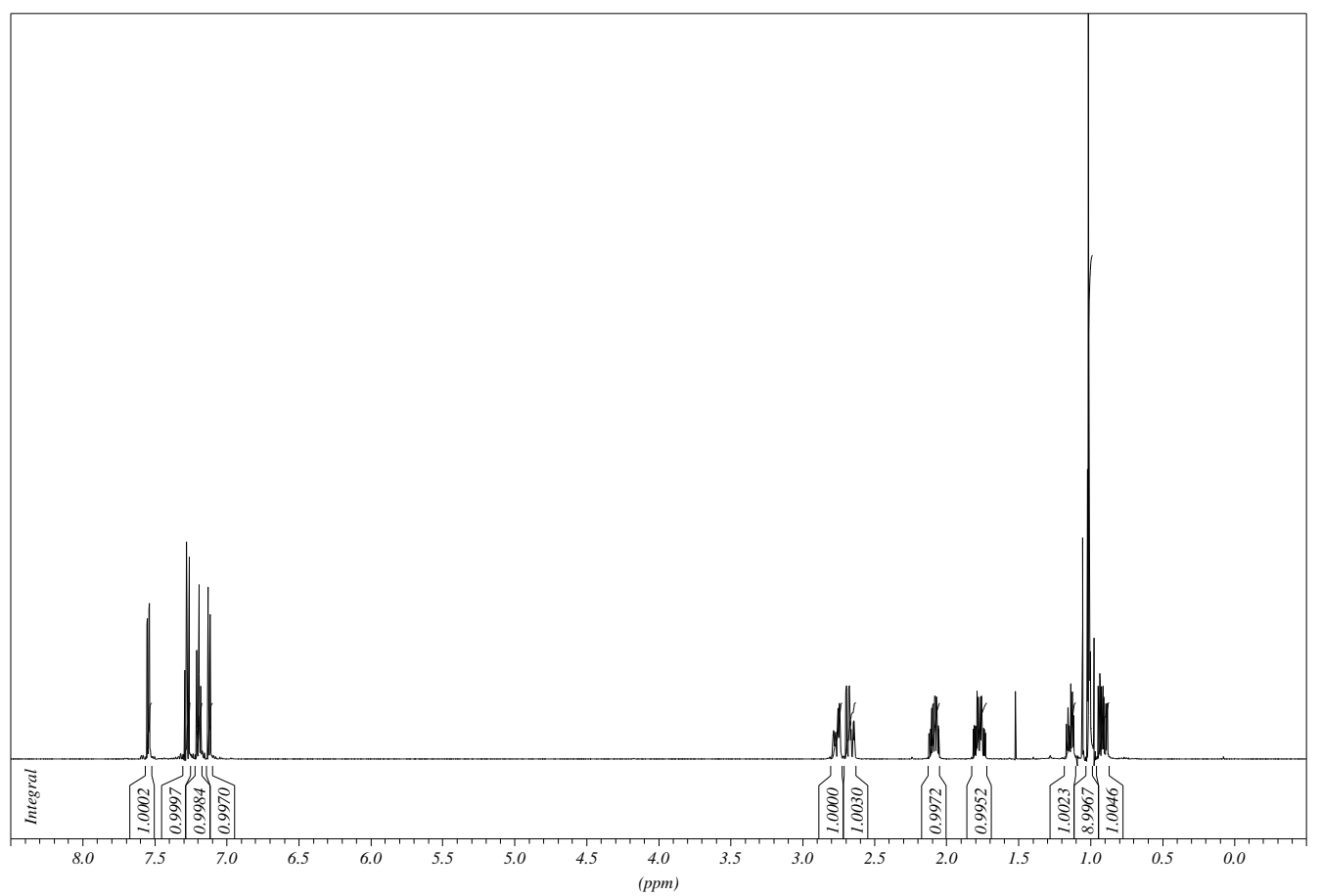


REOEAU26-502,RENDLER,SR311A,CDCL3=77,1
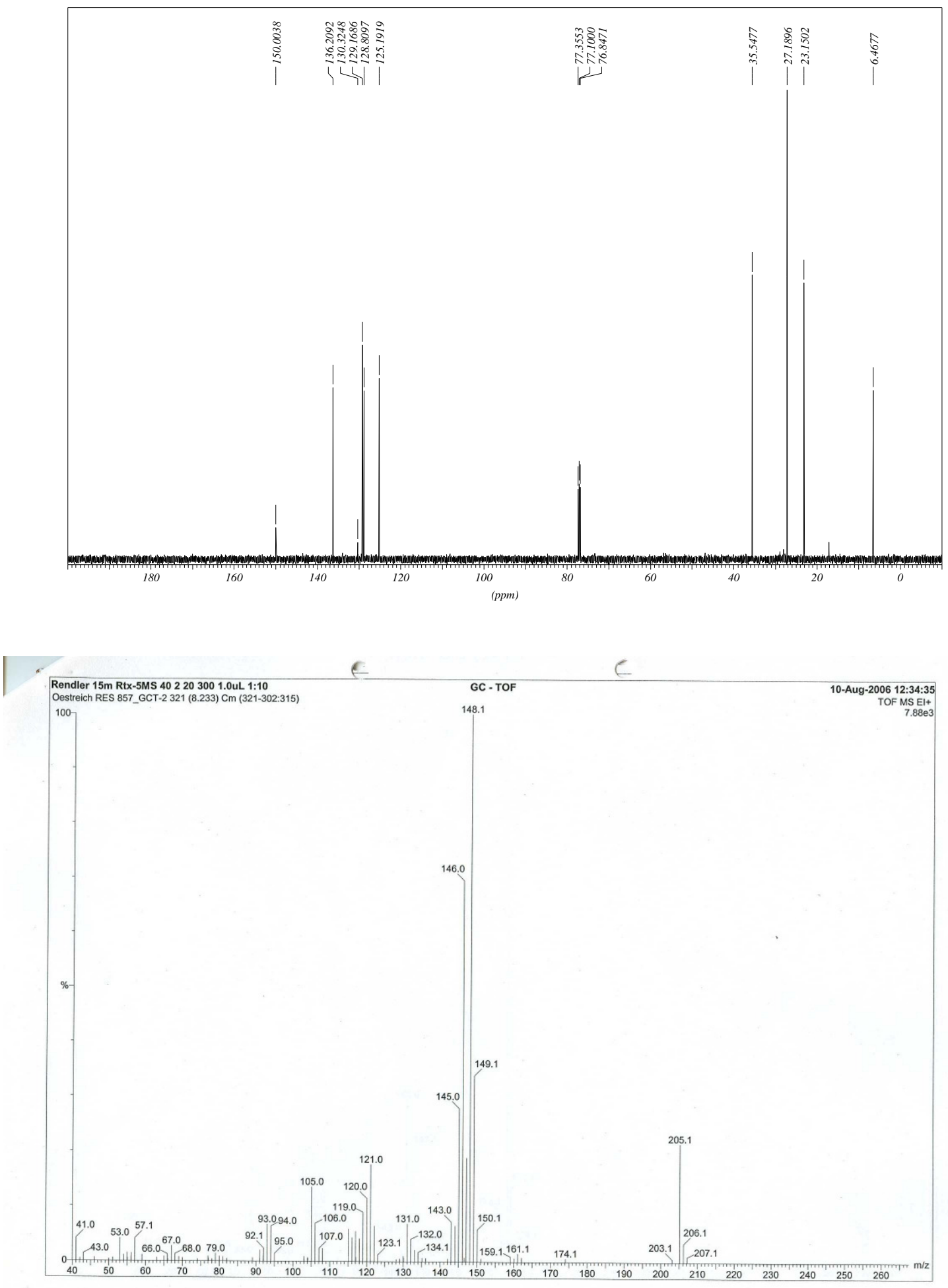


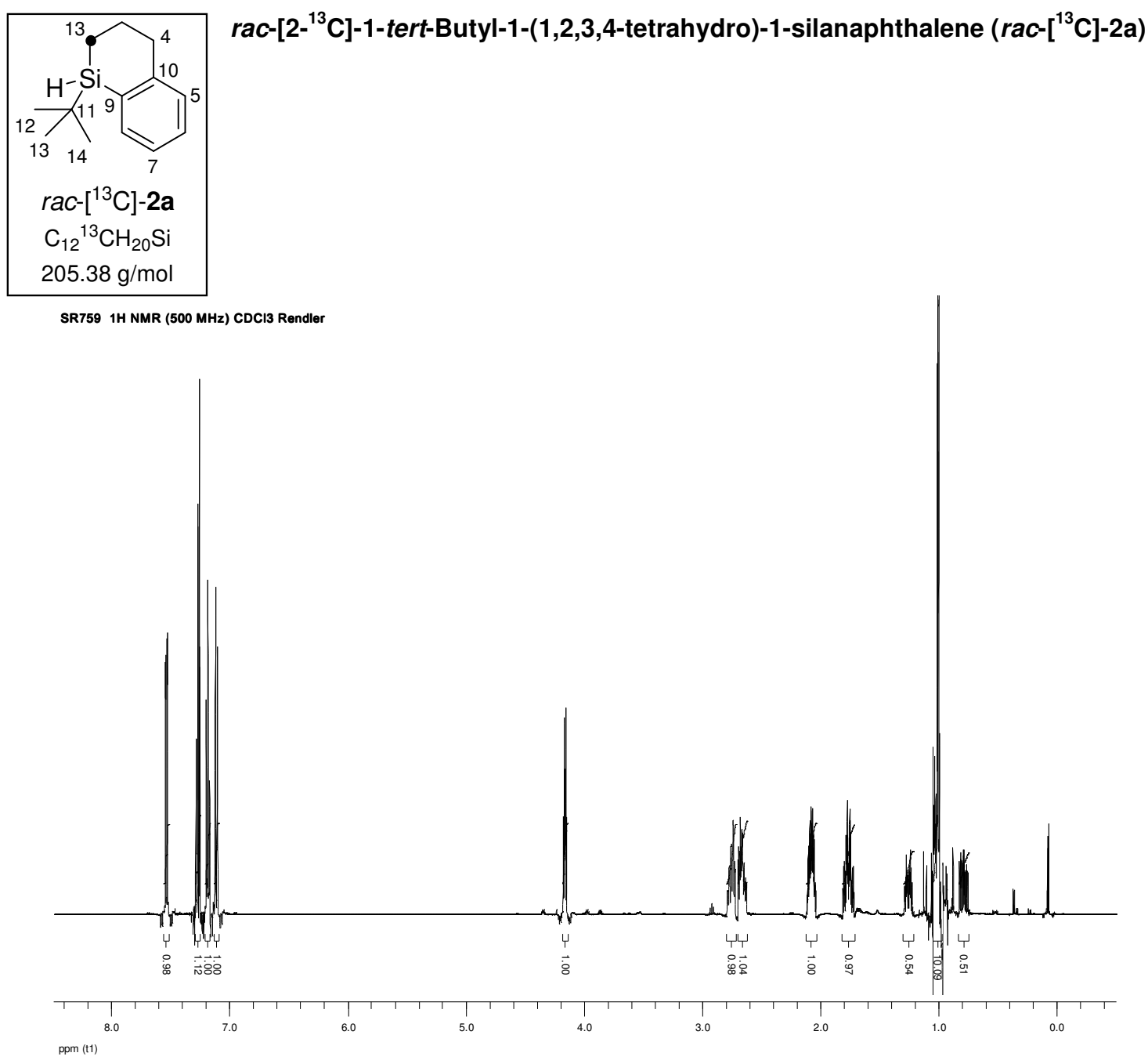


SR759 13C NMR (125 MHz) CDCl3 Rendler

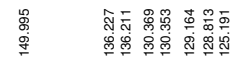

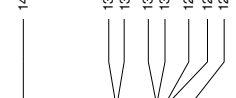
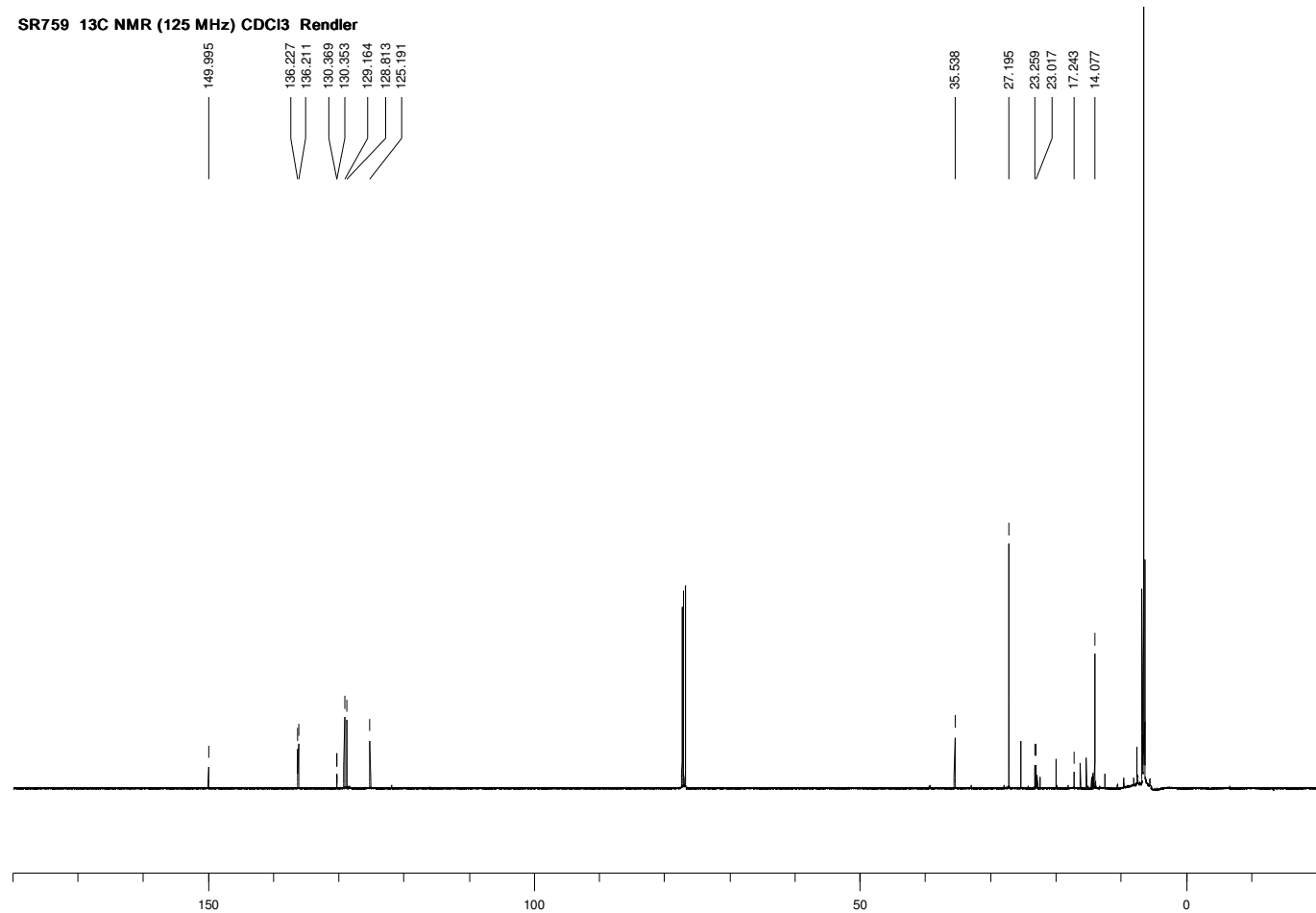

ppm (t1)

100

Tm Rtx-5MS 402203001.0011 1:10

Im Rtx-5MS 402203001.041 1:10

Im Rtx-5MS 40220300 1.0ul 1:10
n RES 759_GCT-2 321 (8.232) Cm (321-309:315)

|

ded

1

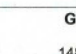

148.2

10-Aug-2006 11:24:24

TOF MSEI+

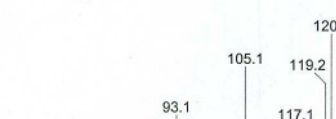

120.2

149.2

146.2

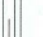

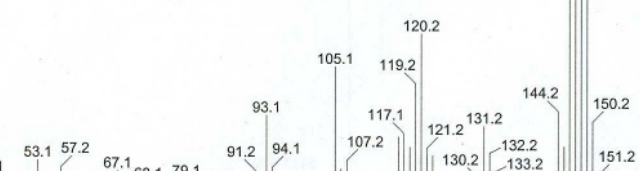

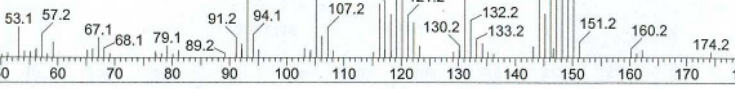

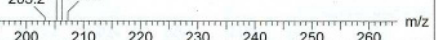

Y la 


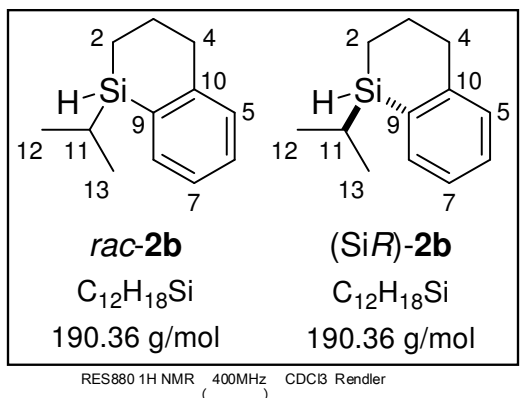

rac-1-Isopropyl-1-(1,2,3,4-tetrahydro)-1-silanaphthalene (rac2b) and

(SiR)-1-Isopropyl-1-(1,2,3,4-tetrahydro)-1-silanaphthalene (rac-2b)

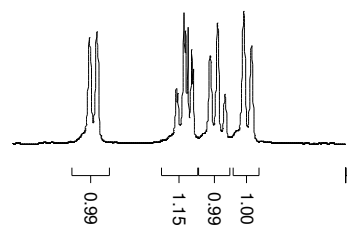

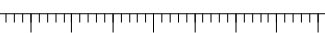
$\begin{array}{lllllll}7.60 & 7.50 & 7.40 & 7.30 & 7.20 & 7.10 & 7.00\end{array}$ गpm (11)
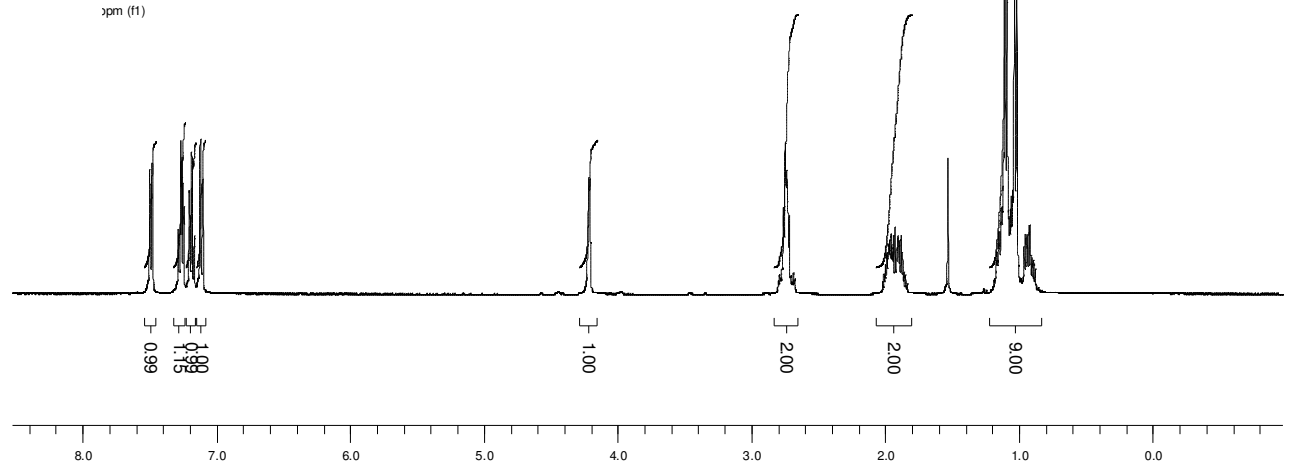
ppm (11)

RES880 13C NMR $100 \mathrm{MHz}$ CDCl3 Rendler
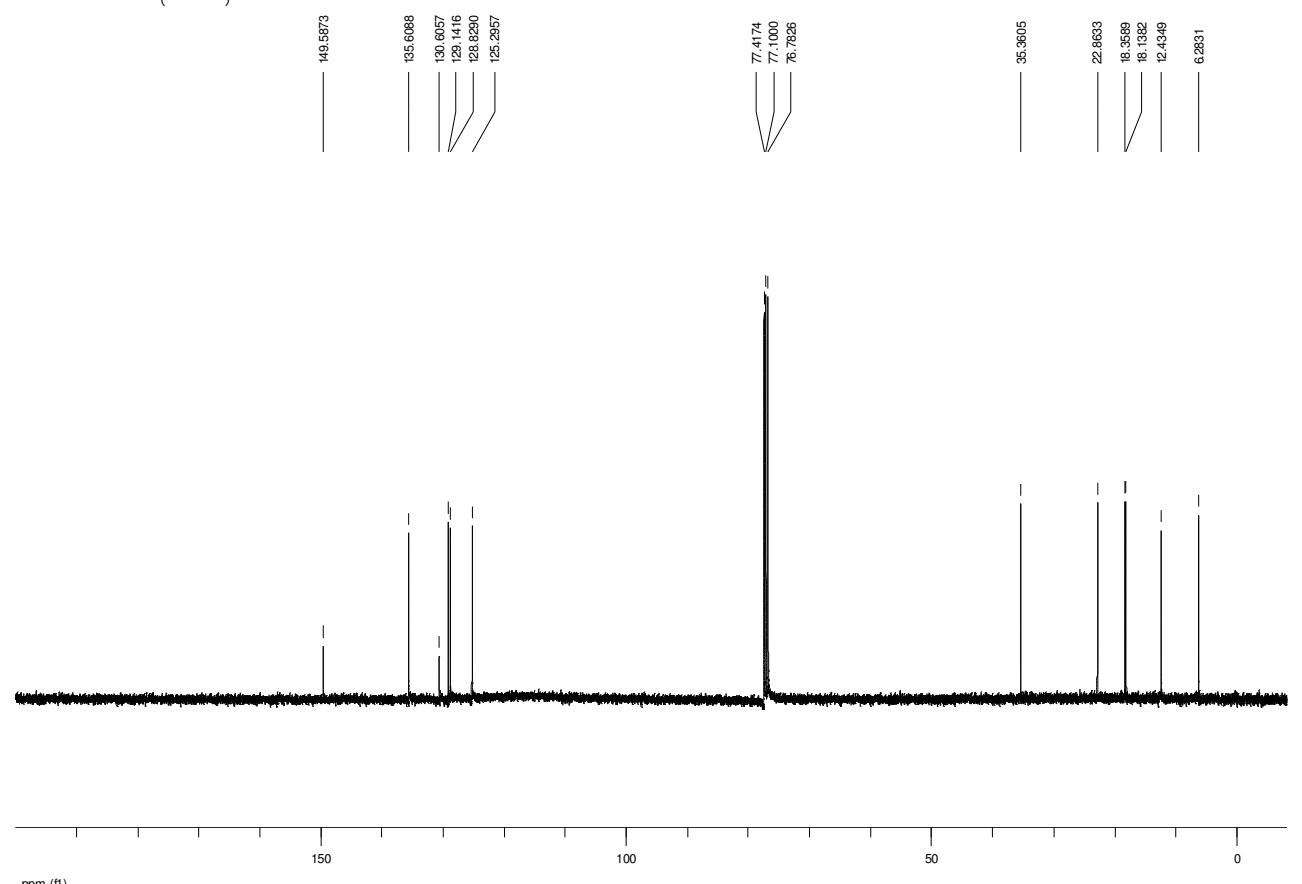


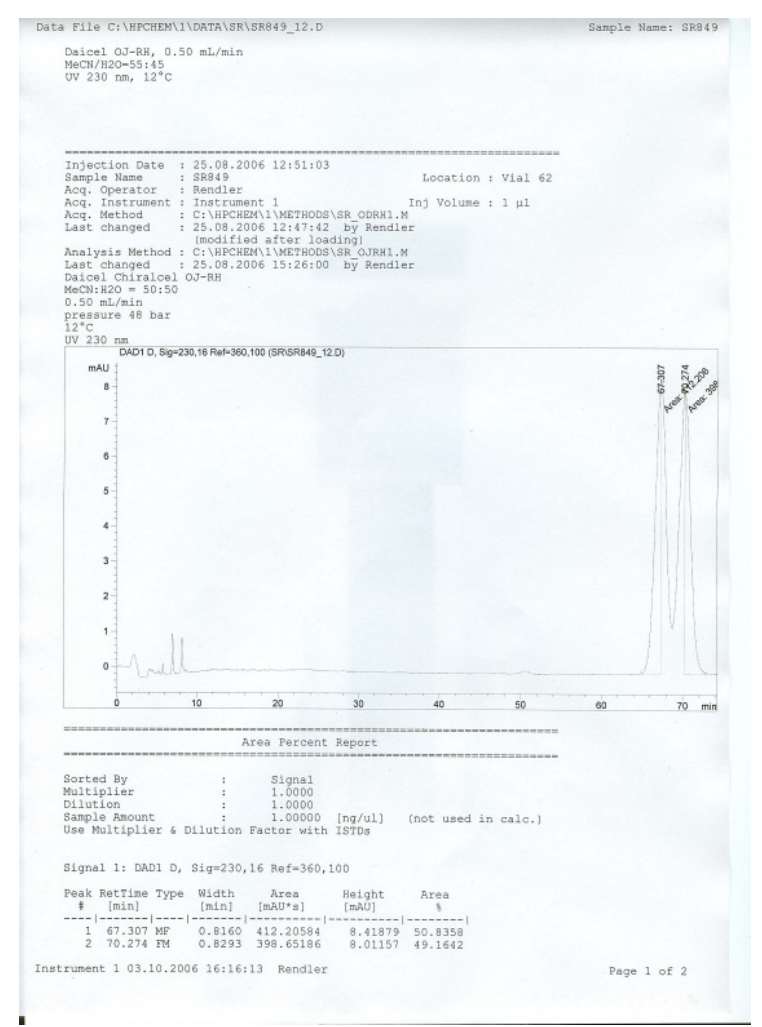

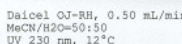

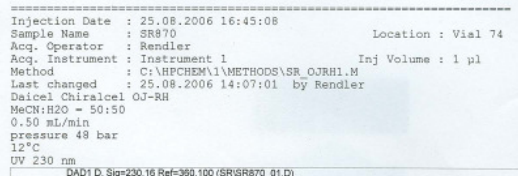

(1) 230 nim

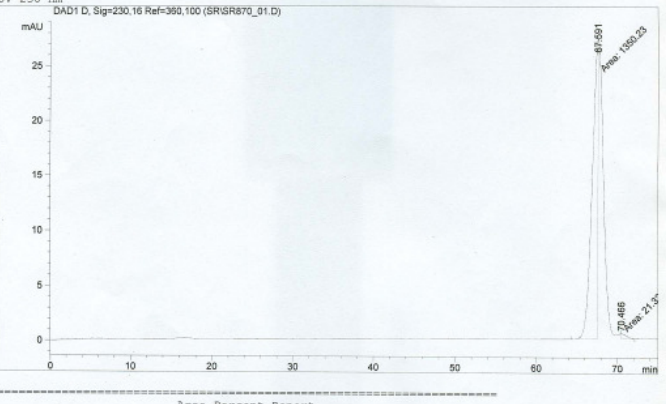

Area Percent Report

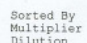

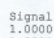

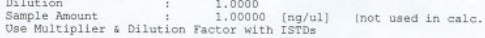

Signa1 1: DAD1 D, Sig-230, 16 Ref-360, 100

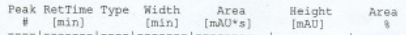

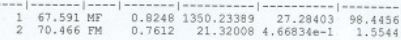

Tota1s:

$\begin{array}{lll}1371.55396 & 27.75096\end{array}$

Enatrument 1 25.08.2006 18:05:04 Rendles

Page 1 of 2

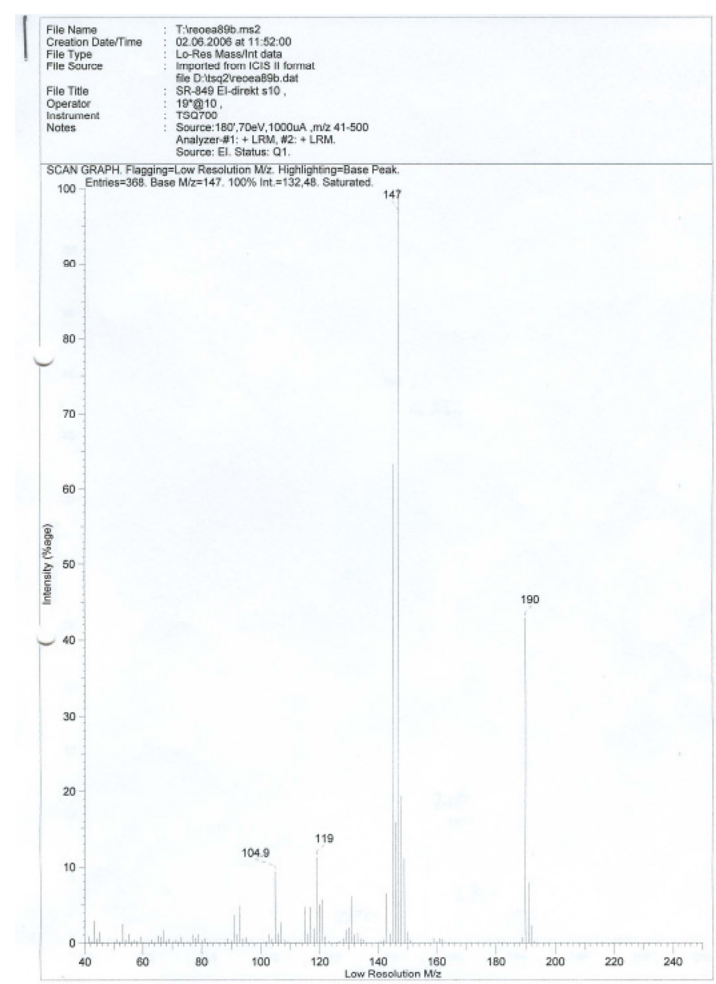




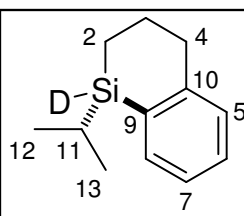

(SiS)-[2H]-2b

$\mathrm{C}_{12} \mathrm{H}_{17} \mathrm{DSi}$

$191.36 \mathrm{~g} / \mathrm{mol}$

(SiS)-[1- $\left.{ }^{2} \mathrm{H}\right]-1-$ Isopropyl-1-(1,2,3,4-tetrahydro)-1-silanaphthalene (SiS)-[ $\left.\left.{ }^{2} \mathrm{H}\right]-2 \mathrm{~b}\right)$
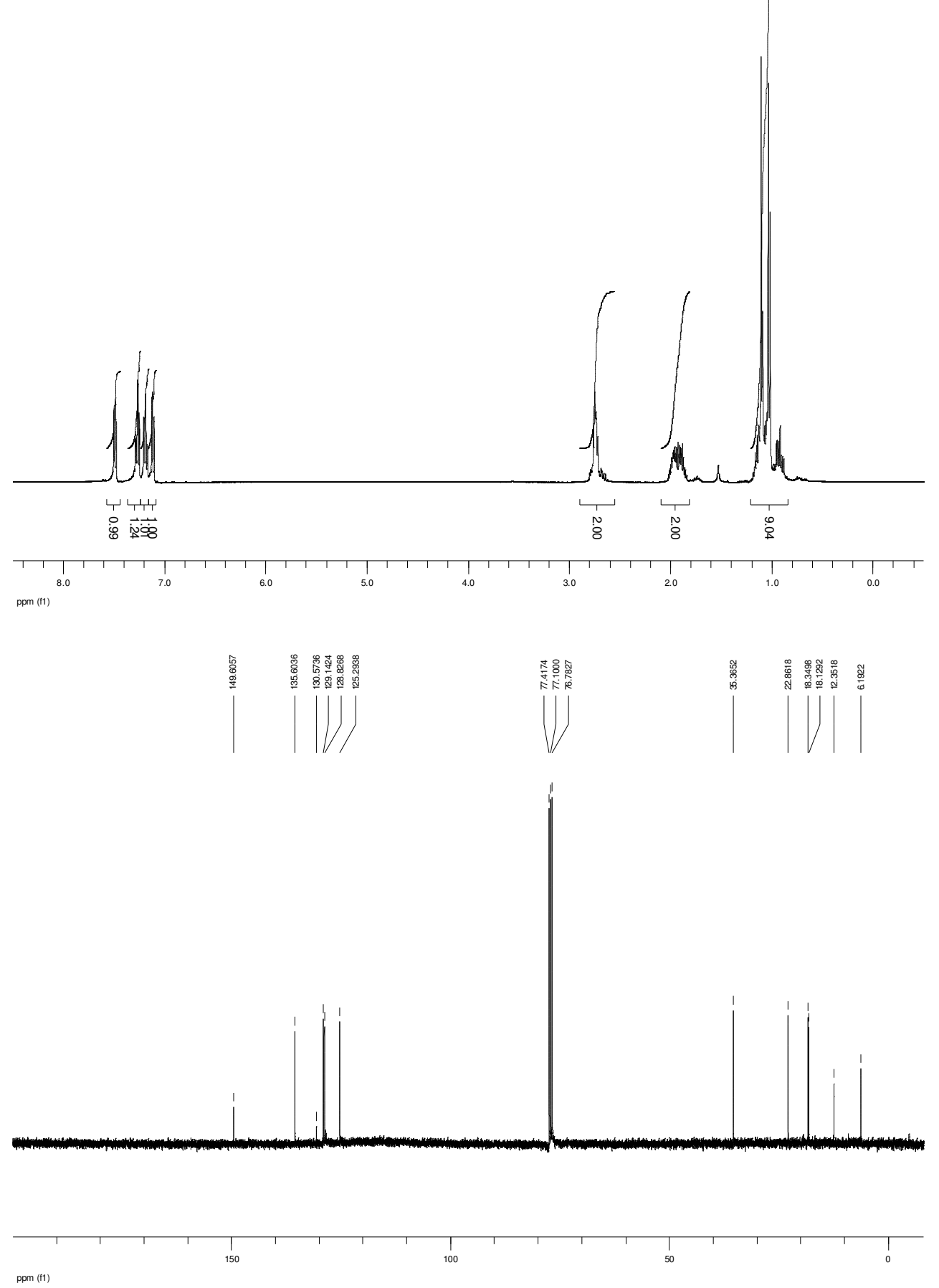

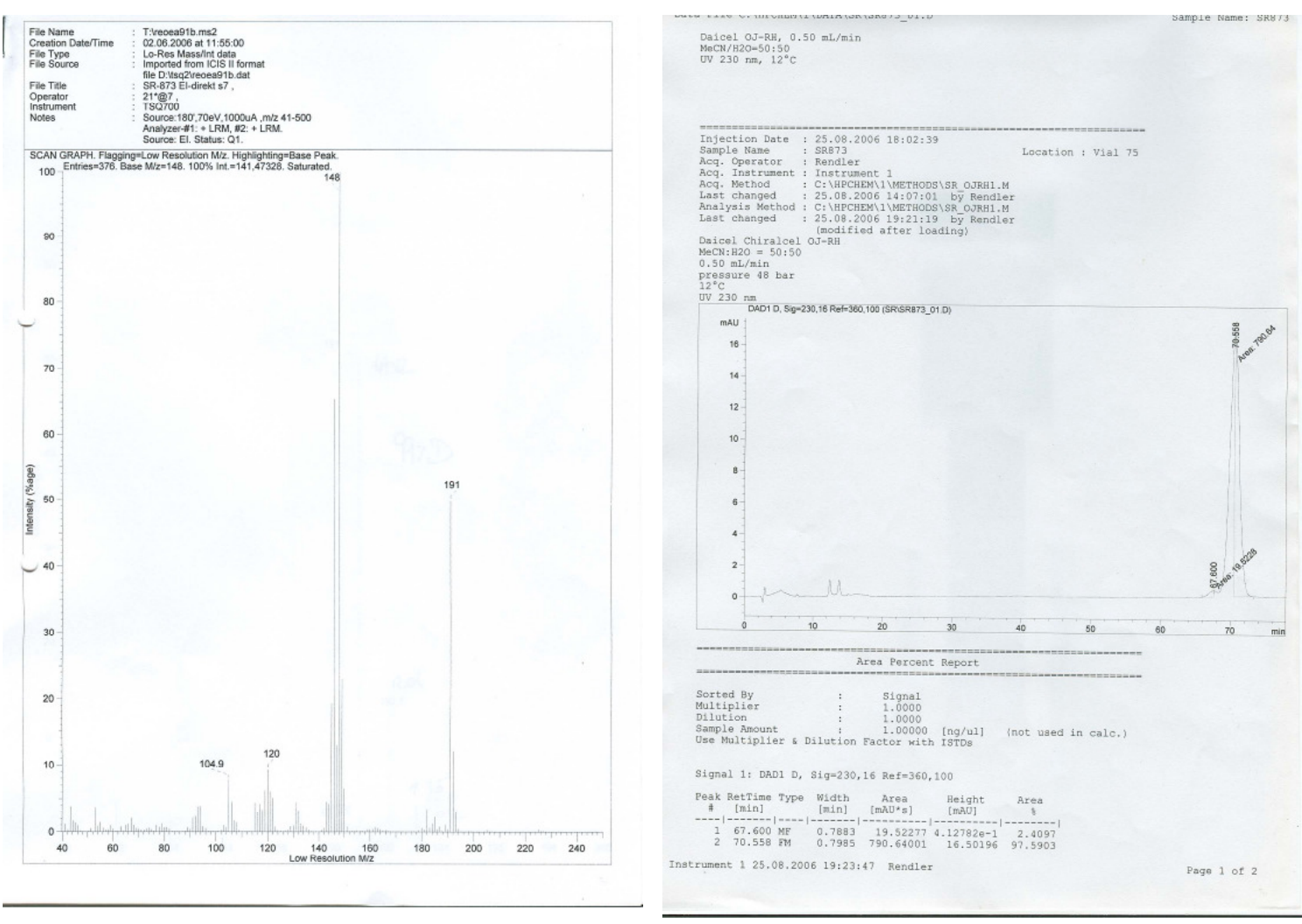

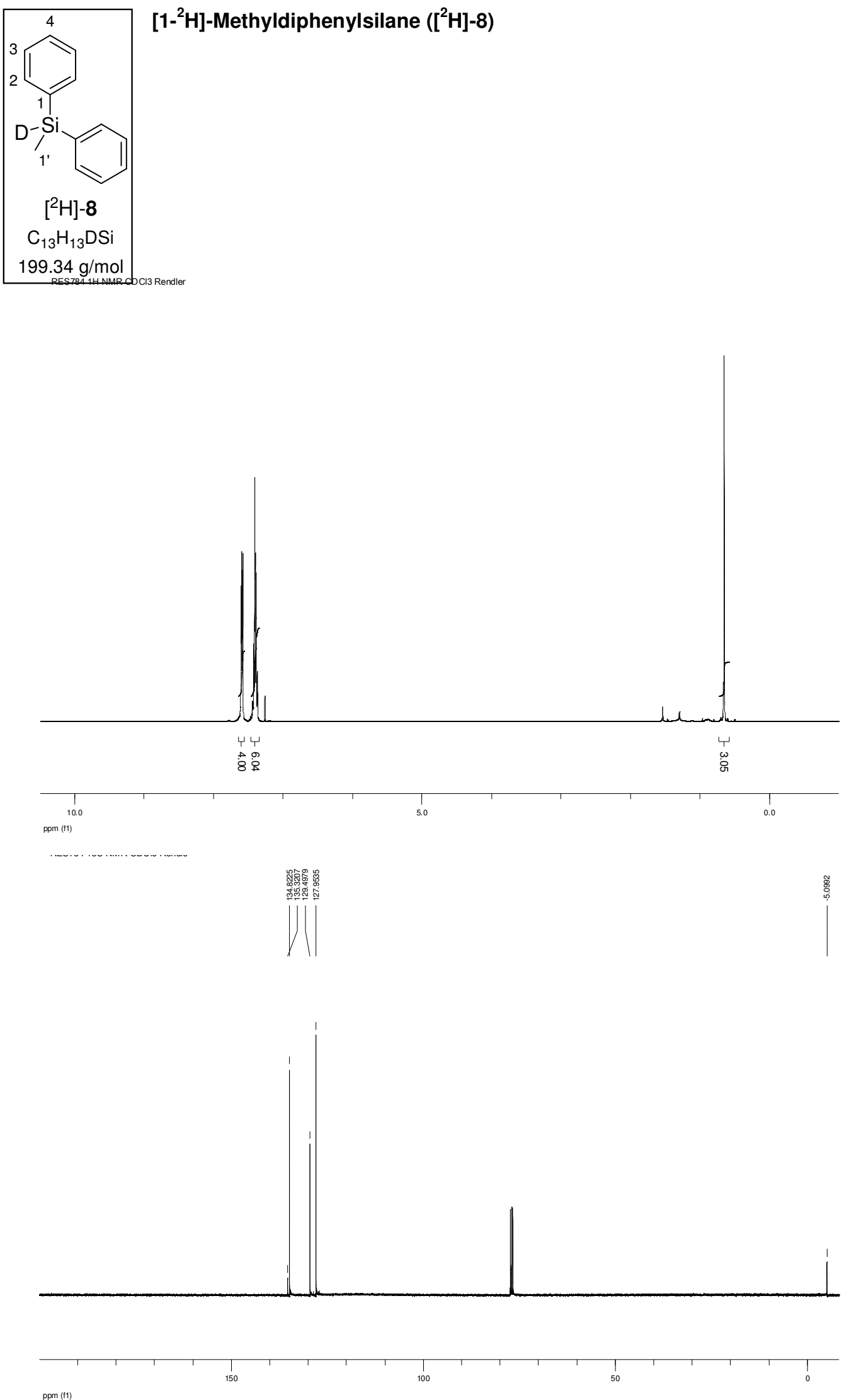
$\left[{ }^{2} \mathrm{H}\right]-8$

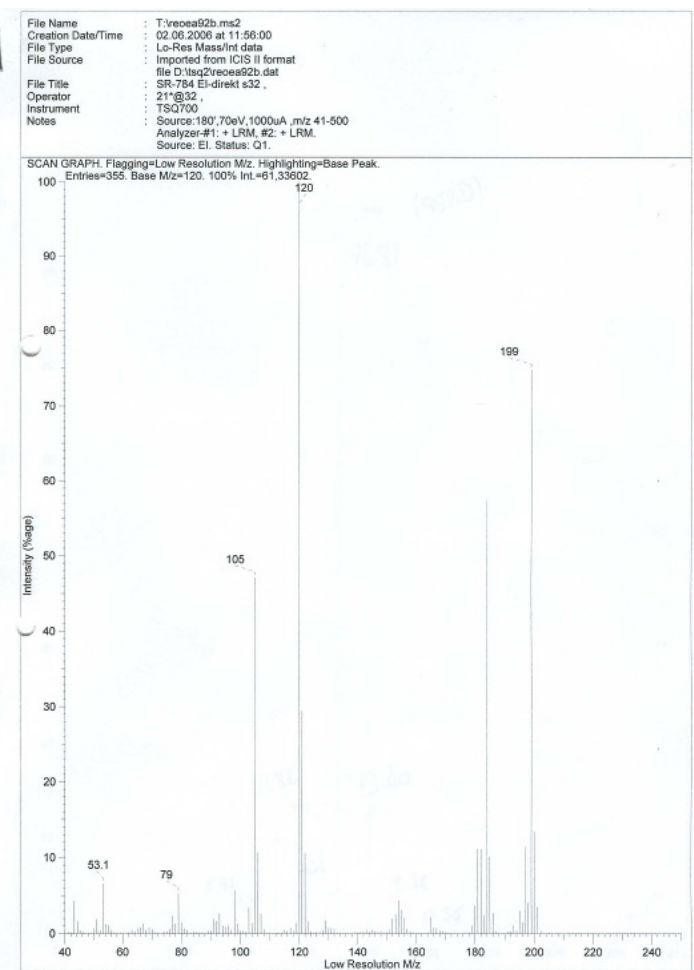

VS. 8

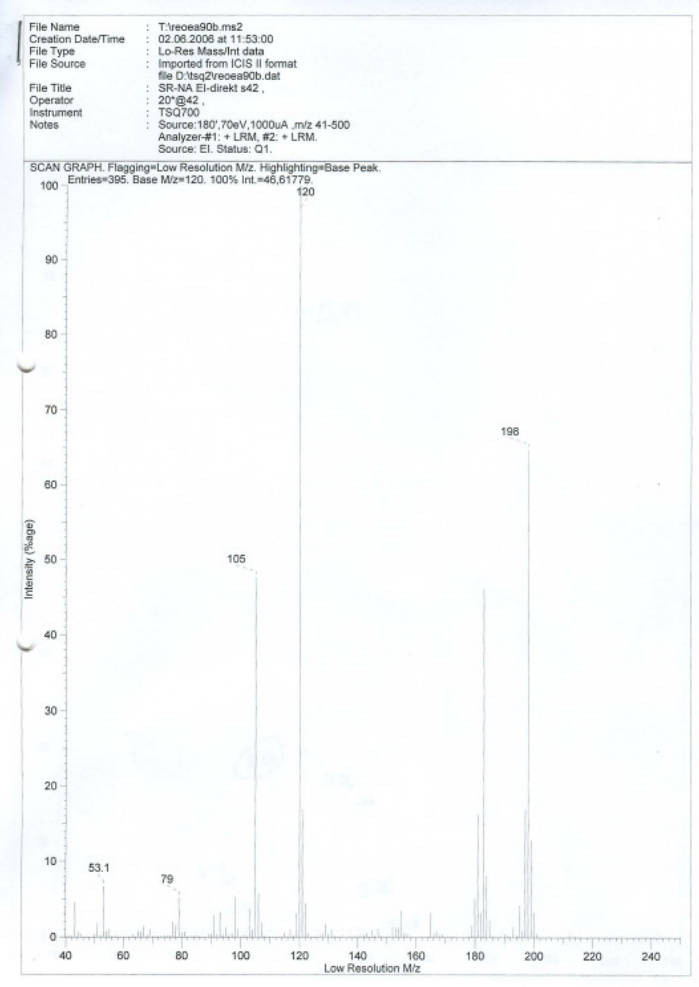




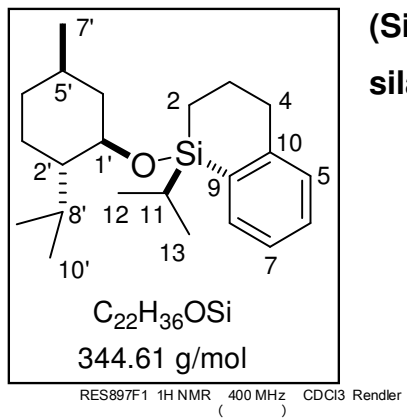

(SiS)-1-Isopropyl-1-[(1R,2S,5R)-1-menthyloxy]-1,2,3,4-tetrahydro-1silanaphthalene

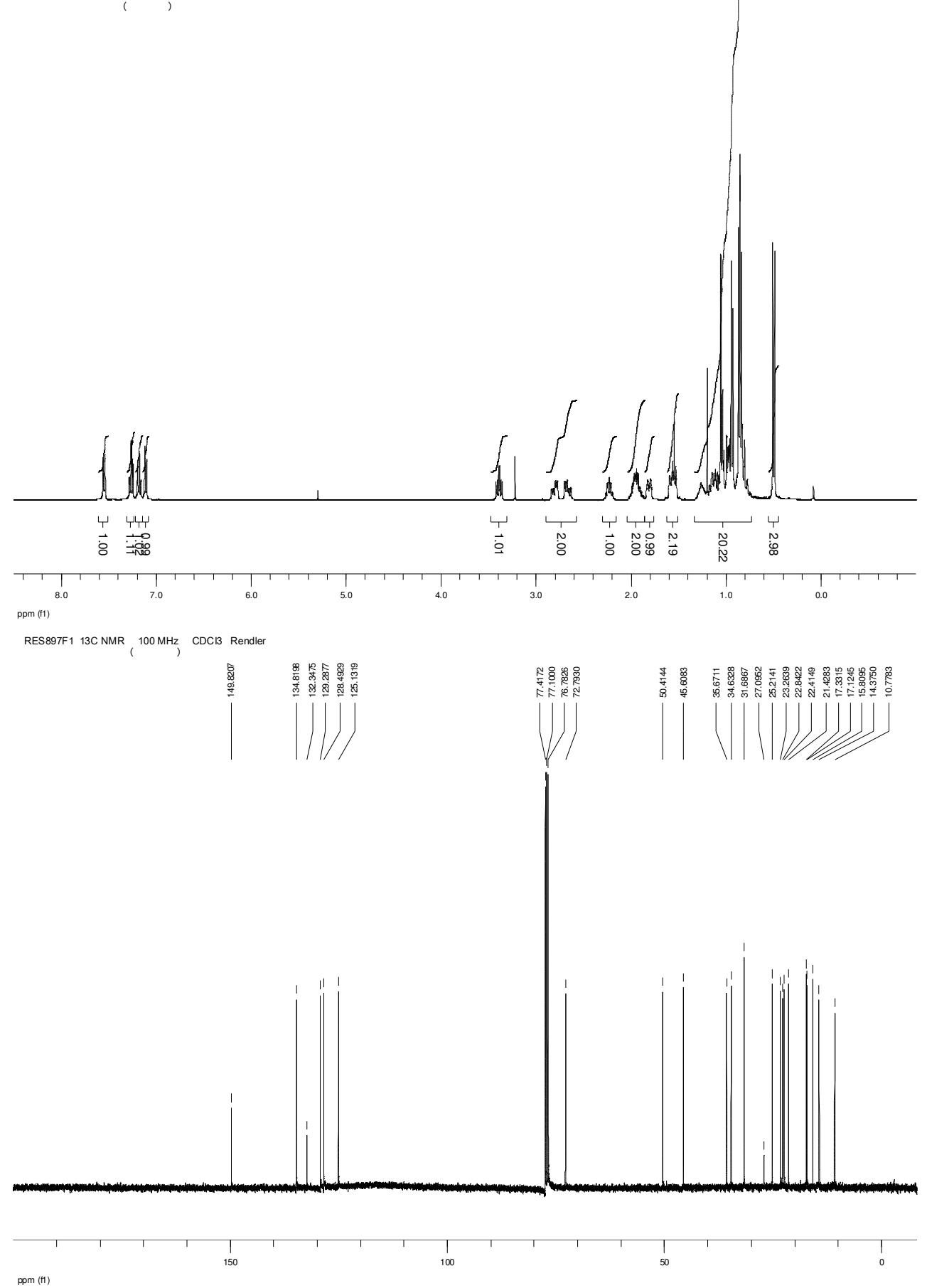




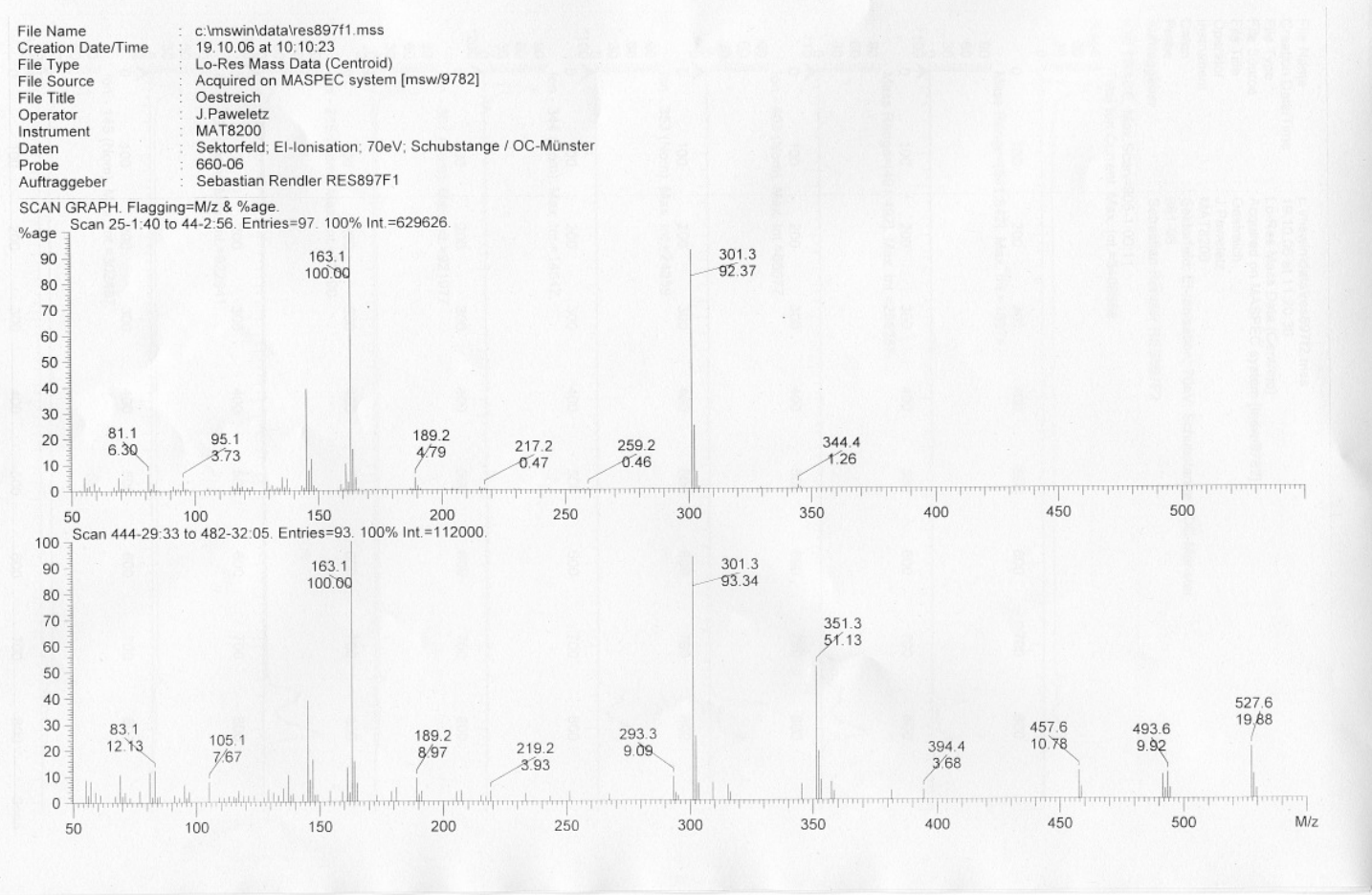




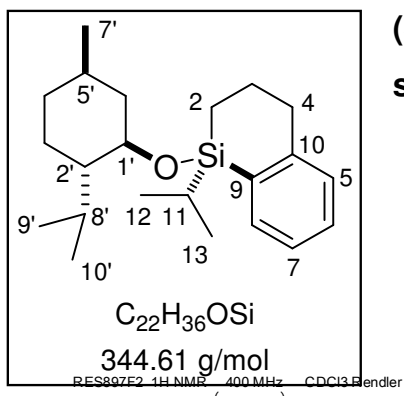

(Si $R$ )-1-Isopropyl-1-[(1R,2S,5R)-1-menthyloxy]-1,2,3,4-tetrahydro-1silanaphthalene

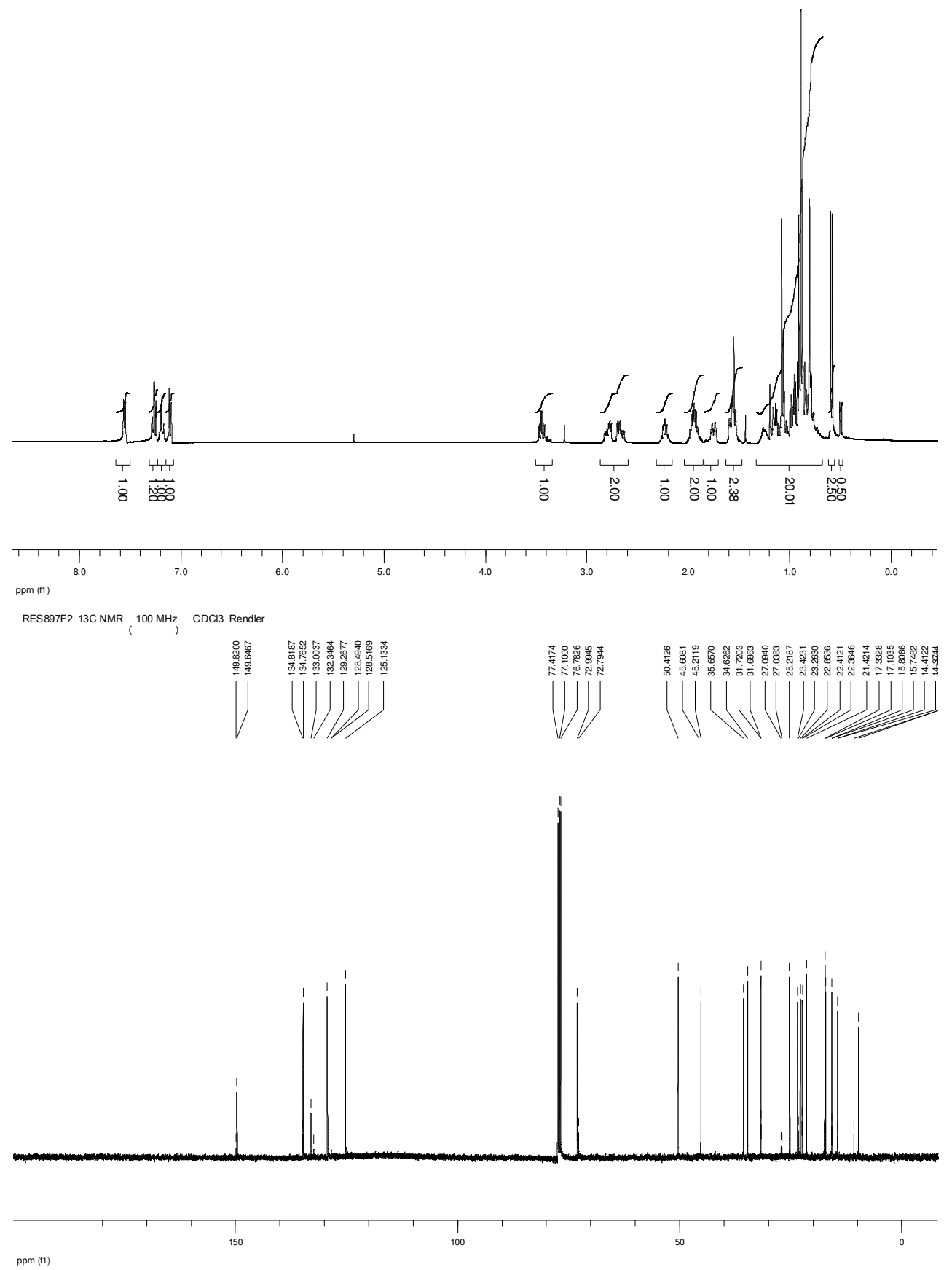




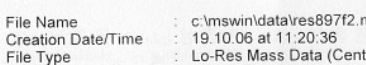

Creation Date/Time : 19.10 .06 at $11.20: 36$

$\begin{array}{ll}\text { File Source } & \text { Acquired on MASPEC system [msw/9782] }\end{array}$

File Title : Oestreich

Operator $\quad J$ Paweletz

Instrument
Daten

SCAN GRAPH. Flagging $=$ M/z $\&$ \%age.
$\%$ age Scan $14-0: 55$ to $27-1: 47$. Entries $=92.100 \%$ Int $=630076$

rage Scan $14-0.55$ to $27-1.47$. Entries $=92.100 \%$ Int $=630076$
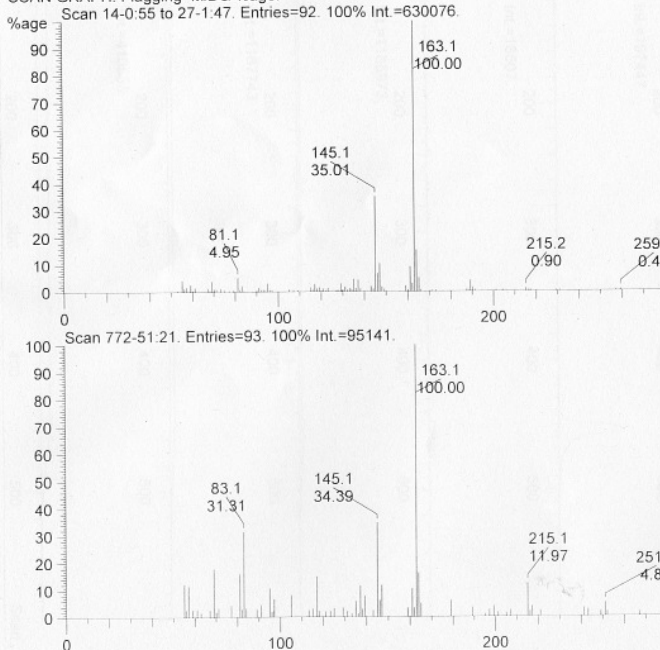

$\mid \begin{aligned} & 3013 \\ & 96.35\end{aligned}$

163.1

300

400

500

491.5
89.09

${ }_{4374}^{4032}$

251.2
$11.97 \quad 4.87$
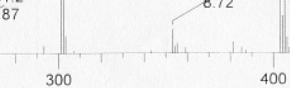

400 


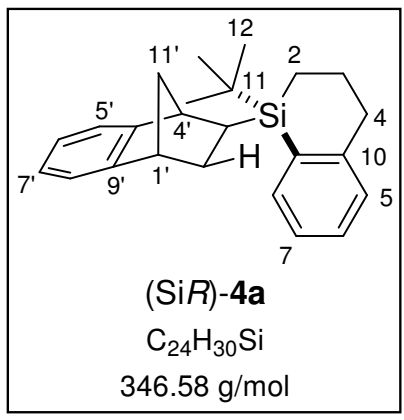

(1S,2S,4R,SiR)-1-tert-Butyl-1-(1,2,3,4-tetrahydro-1,4-methanonaphthalen-2-yl)-1-sila-1,2,3,4-tetrahydronaphthalene [(SiR)-4a]

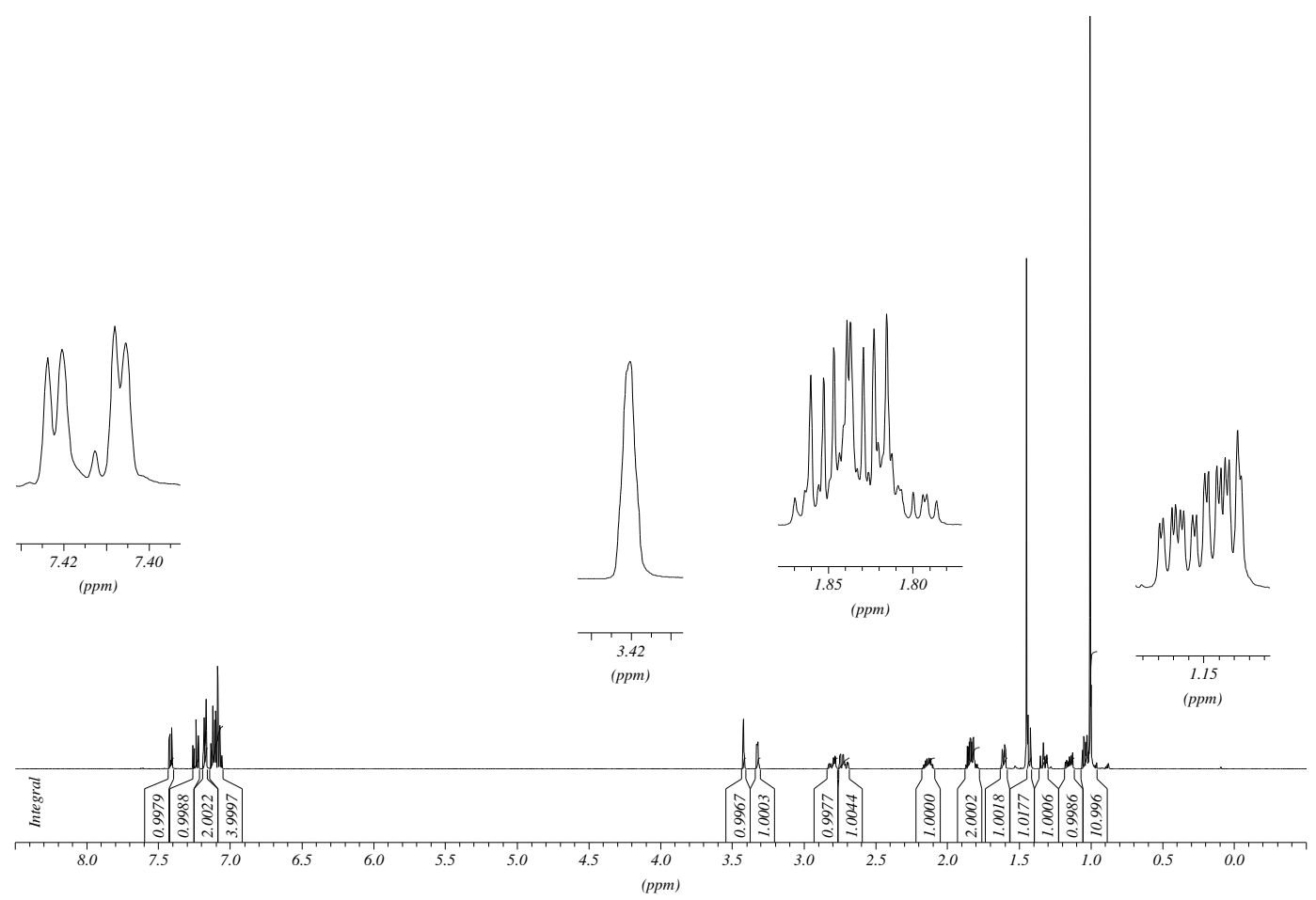



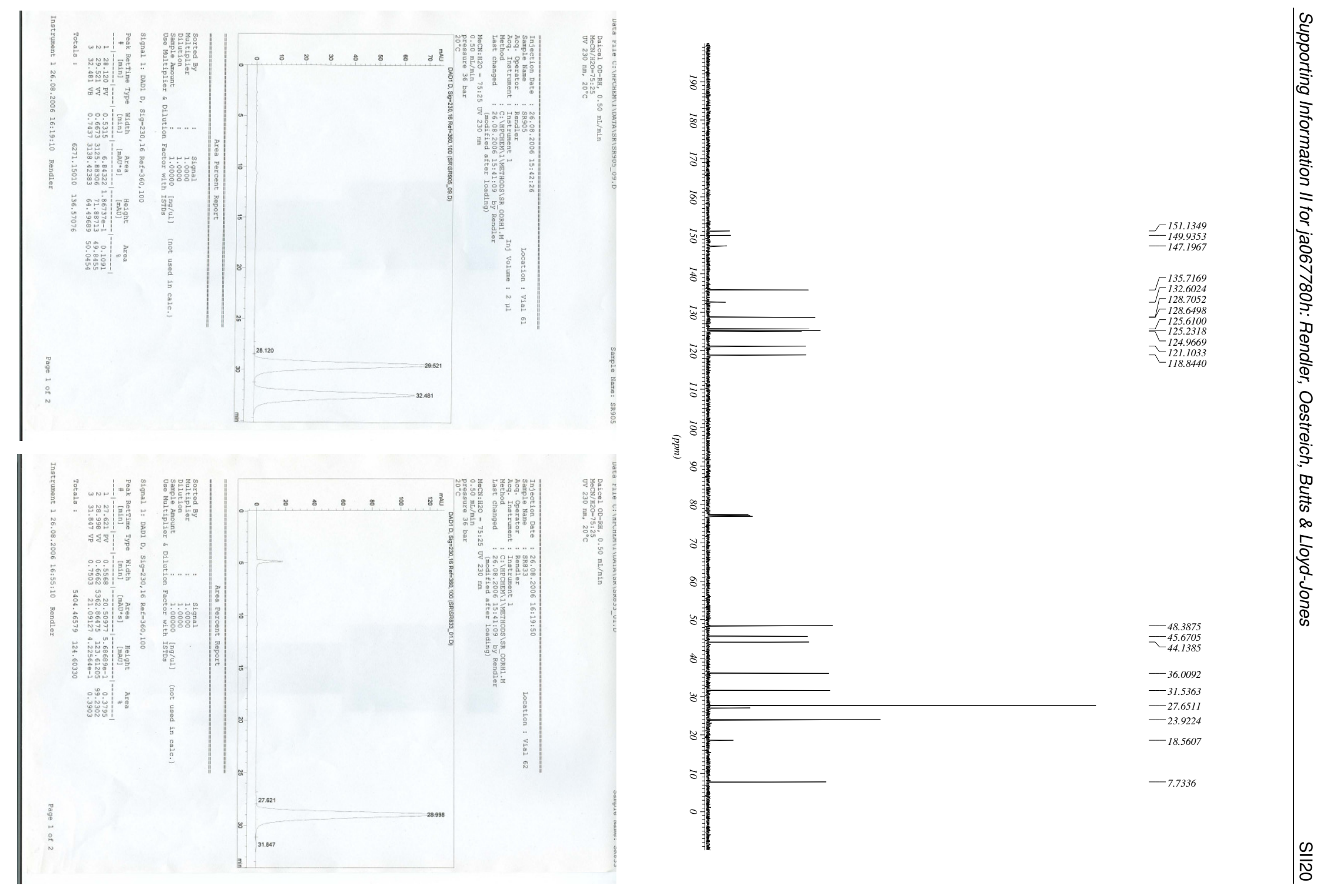


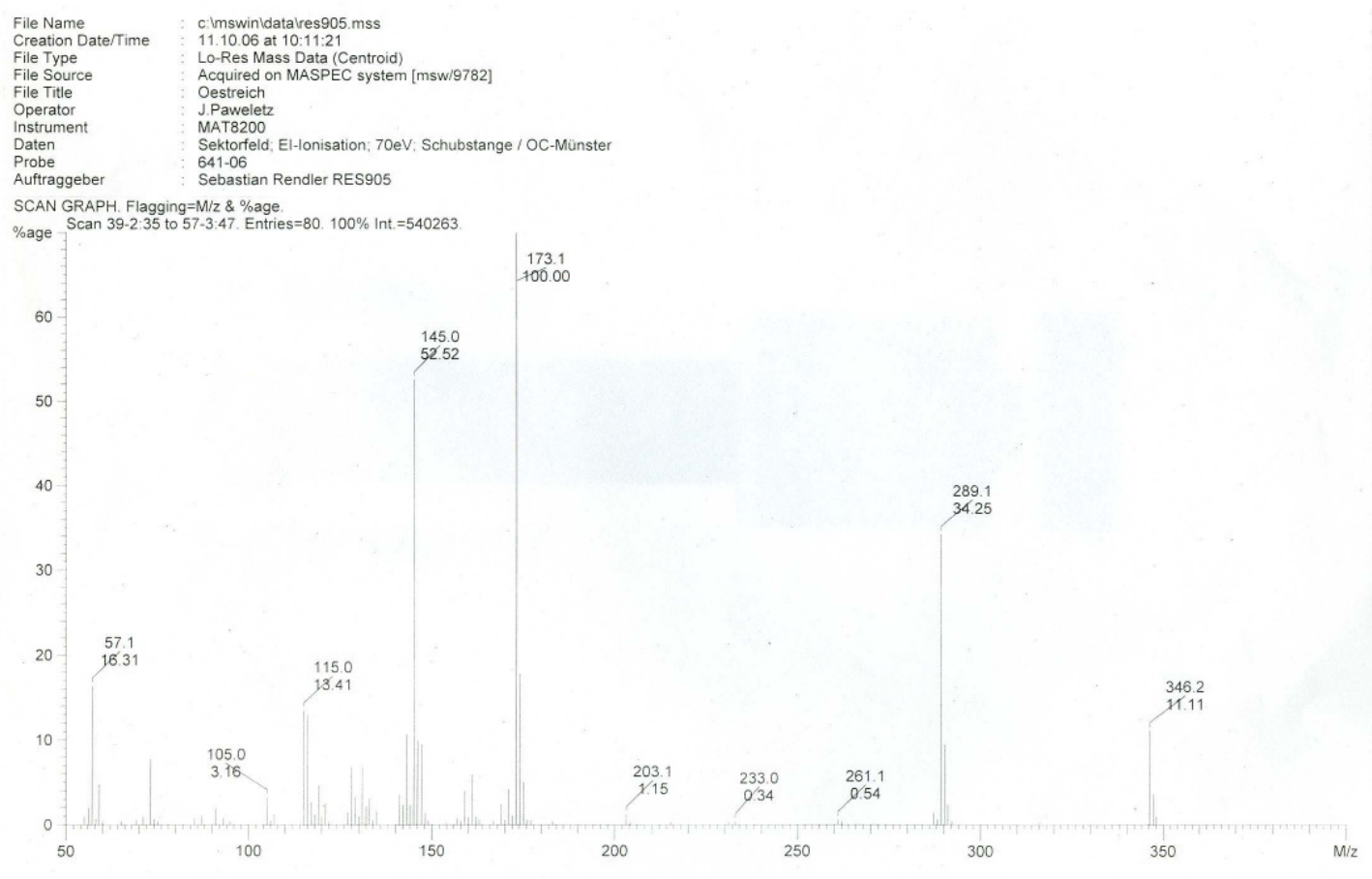




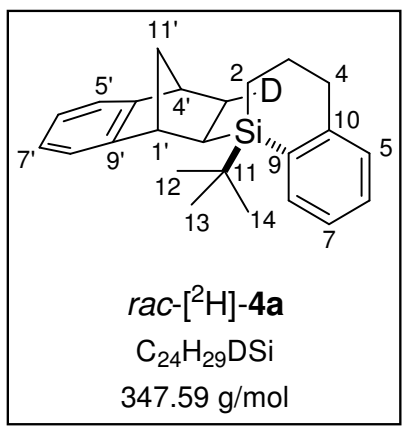

rac-(1 $\left.R^{\star}, 2 R^{\star}, 4 S^{\star}, \mathrm{Si}^{\star}\right)-\left[3^{\prime}-{ }^{2} \mathrm{H}\right]-1-$ tert-Butyl-1-(1,2,3,4-tetrahydro-1,4methano-naphthalen-2-yl)-1-sila-1,2,3,4-tetrahydronaphthalene (rac$\left.\left.\left[{ }^{2} \mathrm{H}\right]-4 \mathrm{a}\right]\right)$

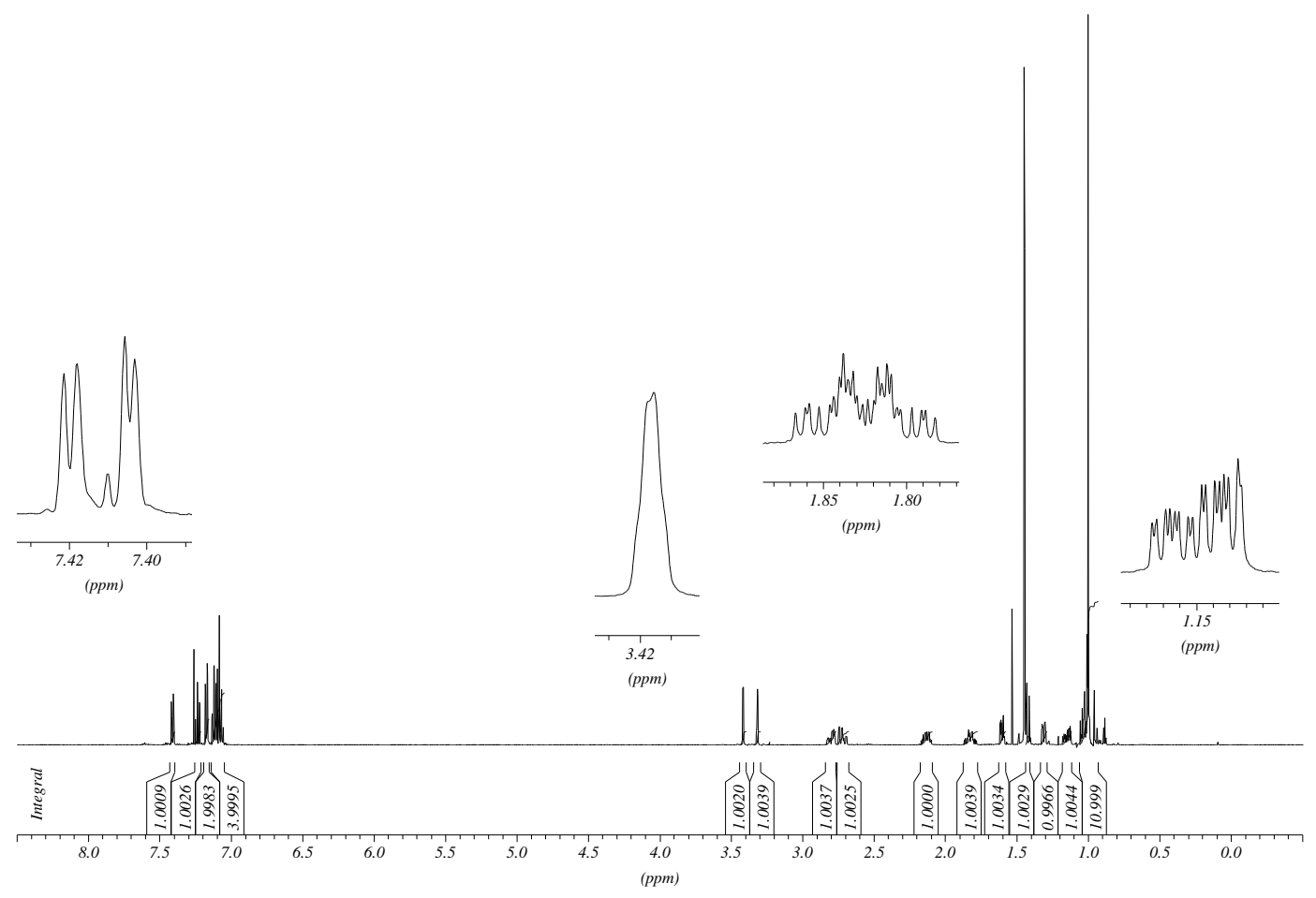



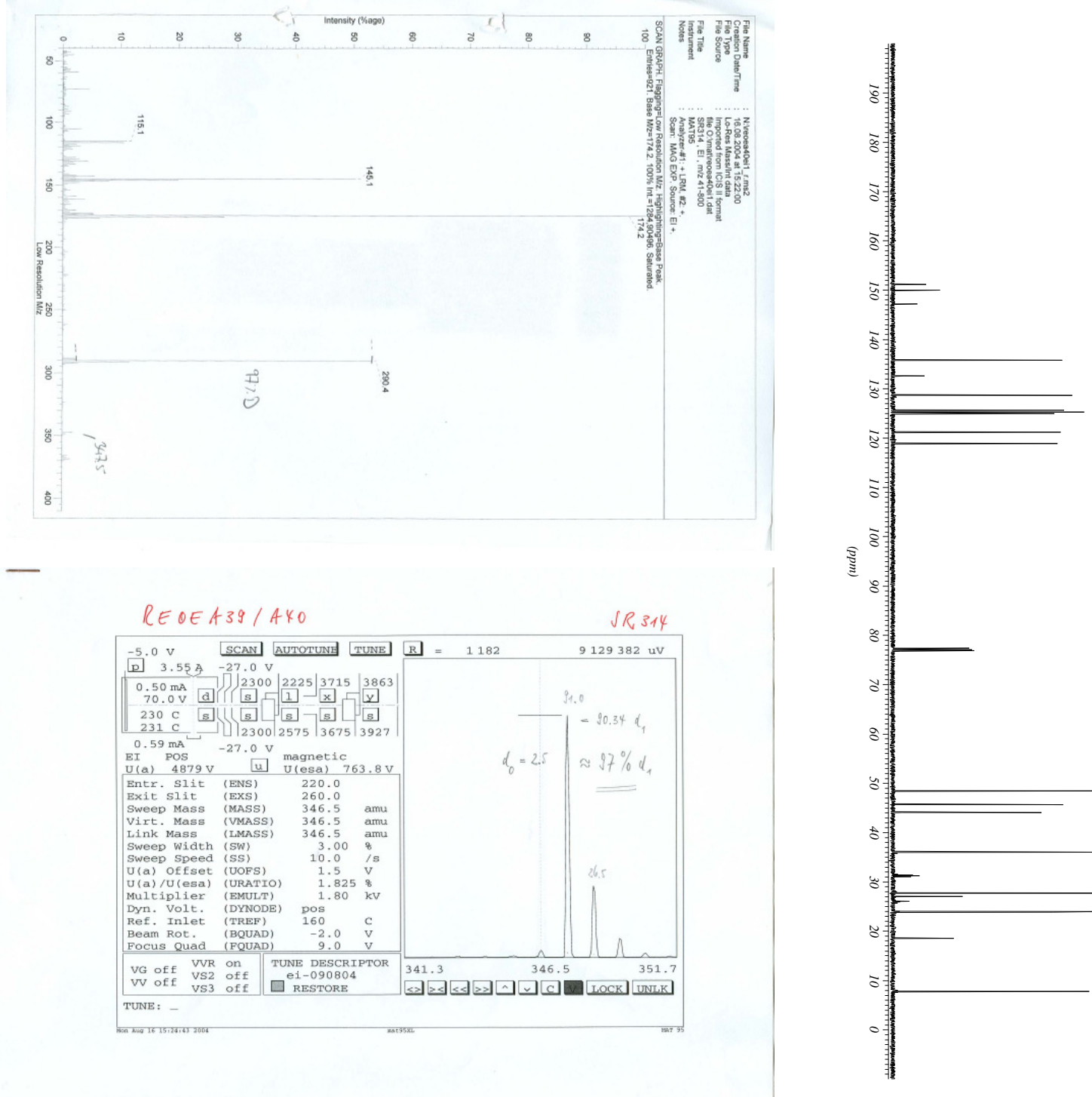


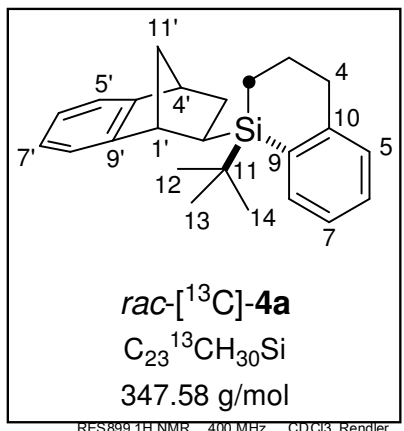

rac- $\left(1 R^{*}, 2 R^{*}, 4 S^{*}, \mathrm{Si}^{*}\right)-\left[2-{ }^{13} \mathrm{C}\right]-1-$ tert-Butyl-1-(1,2,3,4-tetrahydro-1,4methano-naphthalen-2-yl)-1-sila-1,2,3,4-tetrahydronaphthalene [rac$\left.\left[{ }^{13} \mathrm{C}\right]-4 \mathrm{a}\right]$

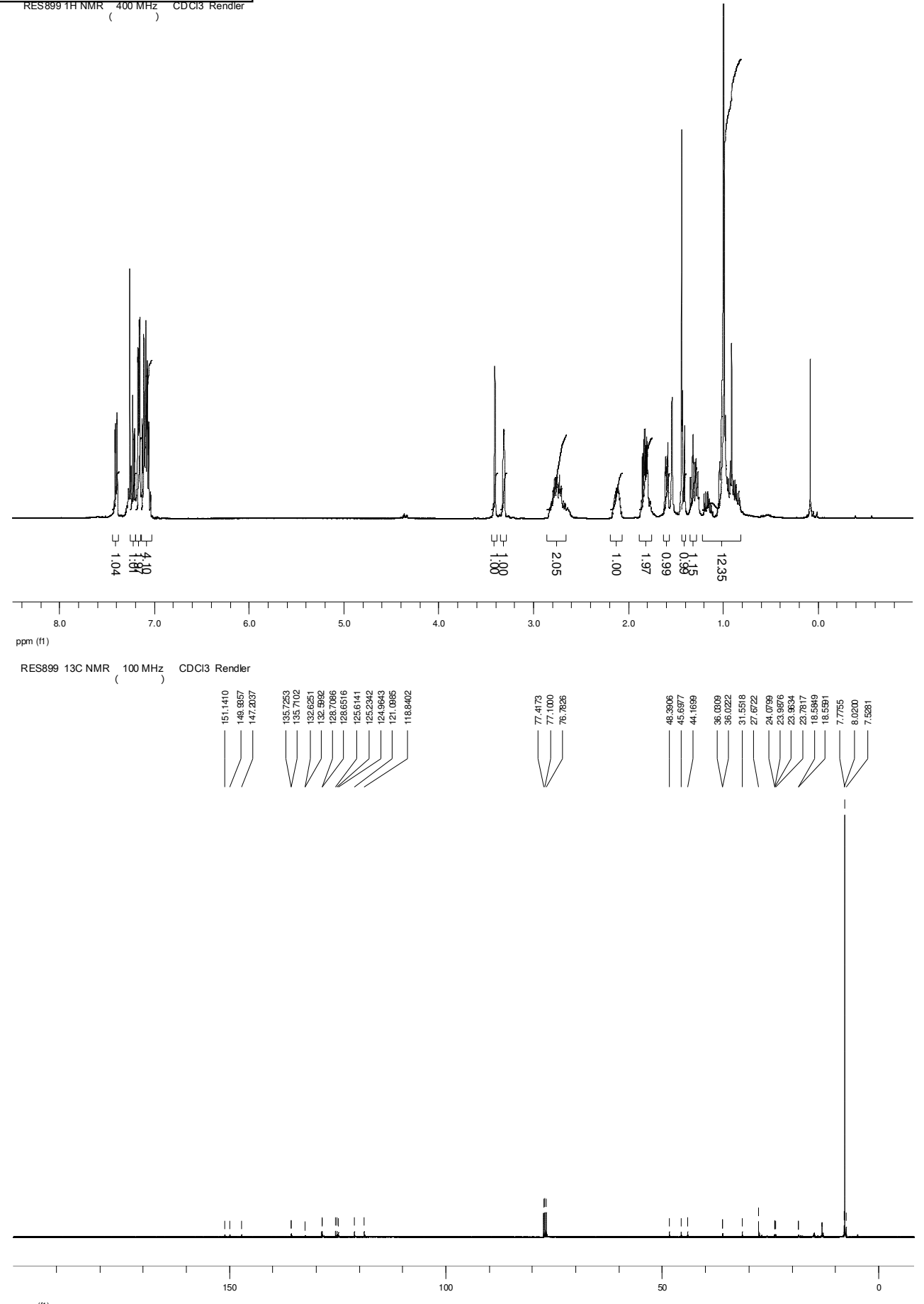




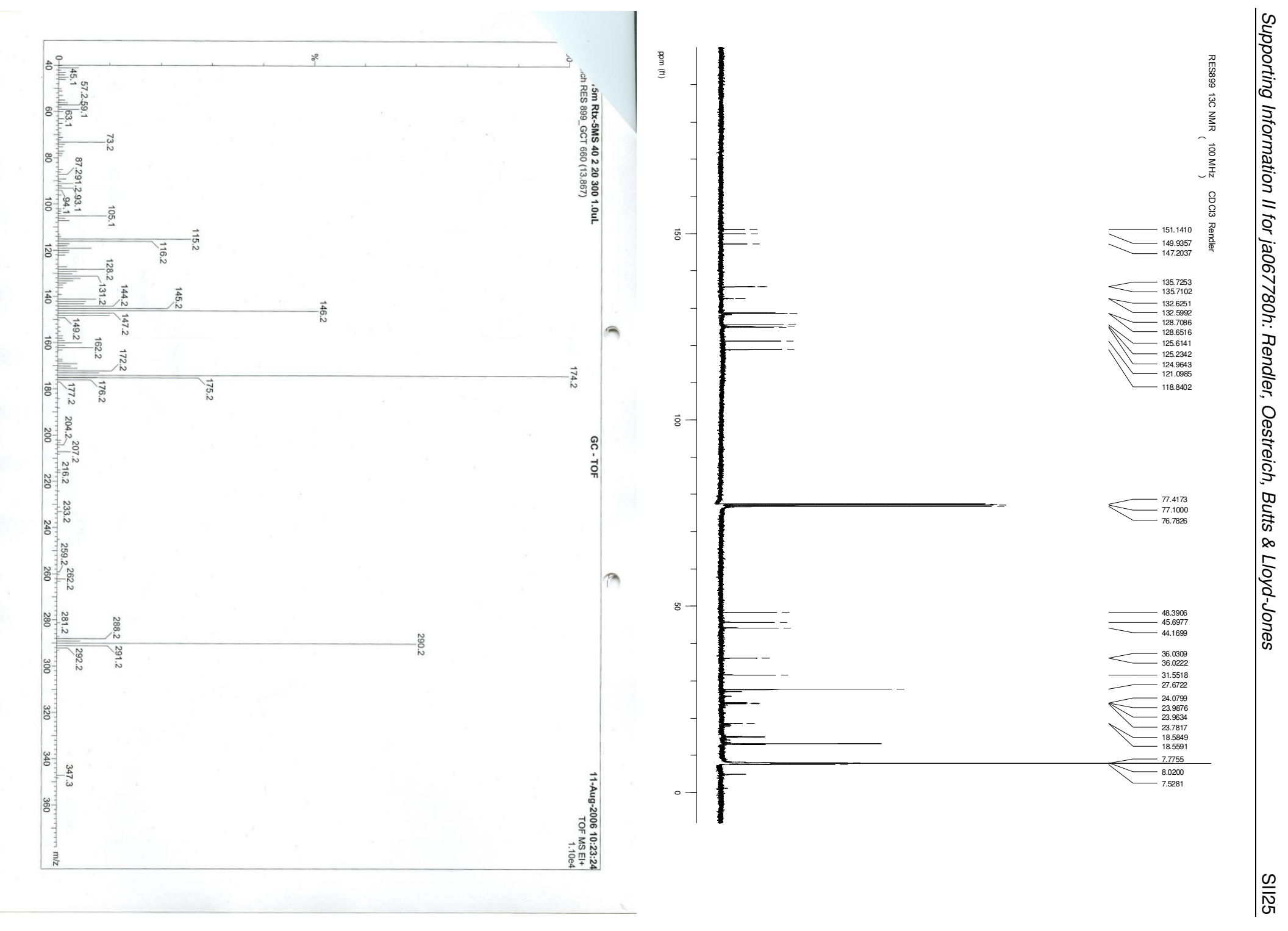




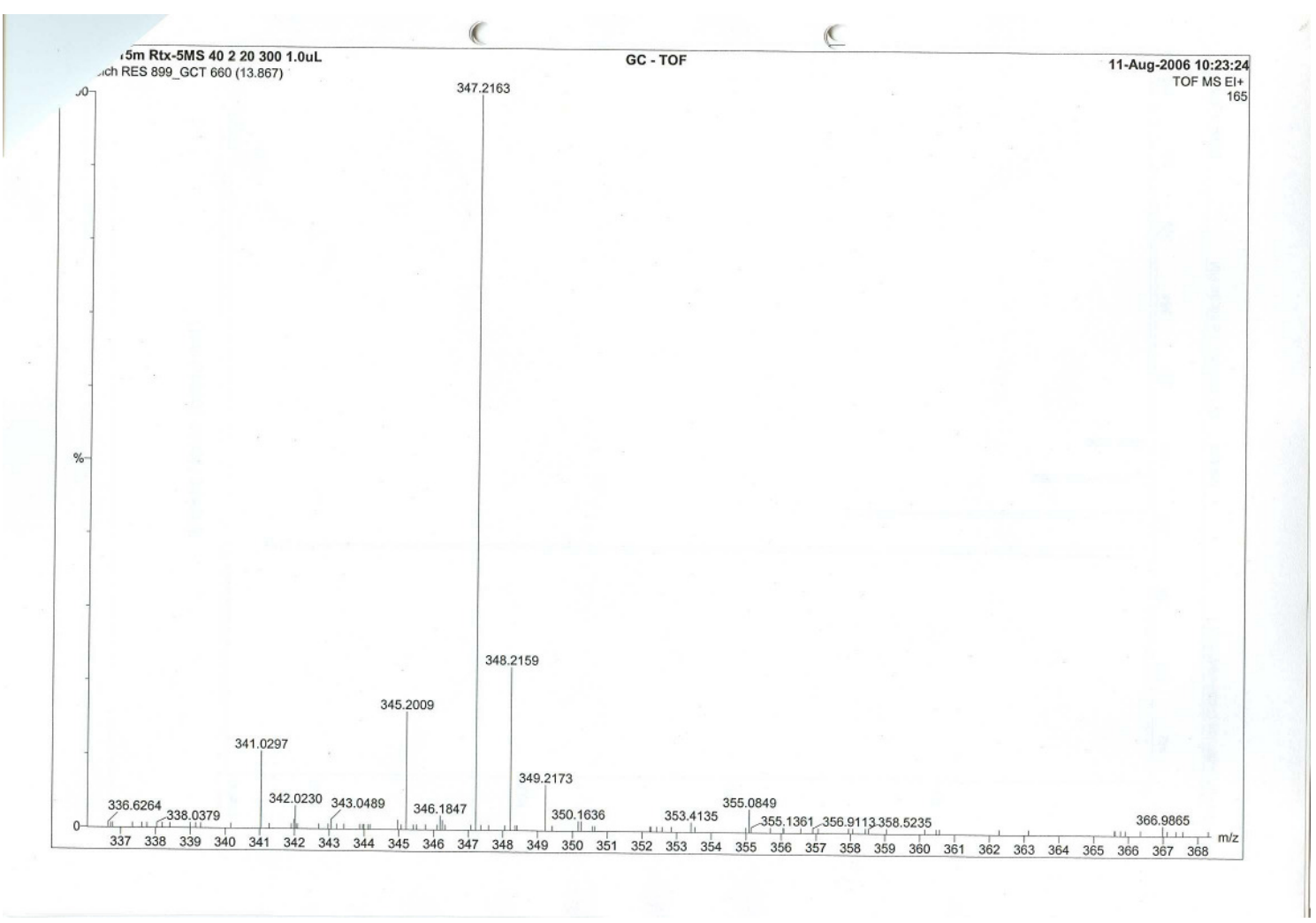




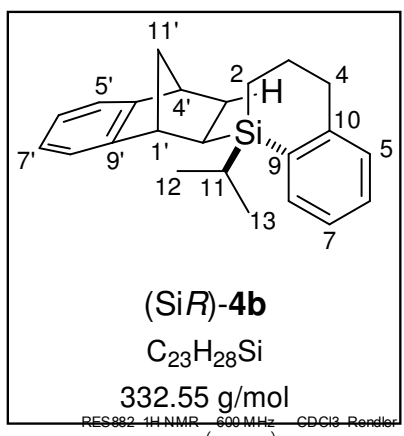

(1R,2R,4S,Si $R$ )-1-Isopropyl-1-(1,2,3,4-tetrahydro-1,4-methanonaphthalen-2-yl)-1-sila-1,2,3,4-tetrahydronaphthalene [(SiR)-4b]
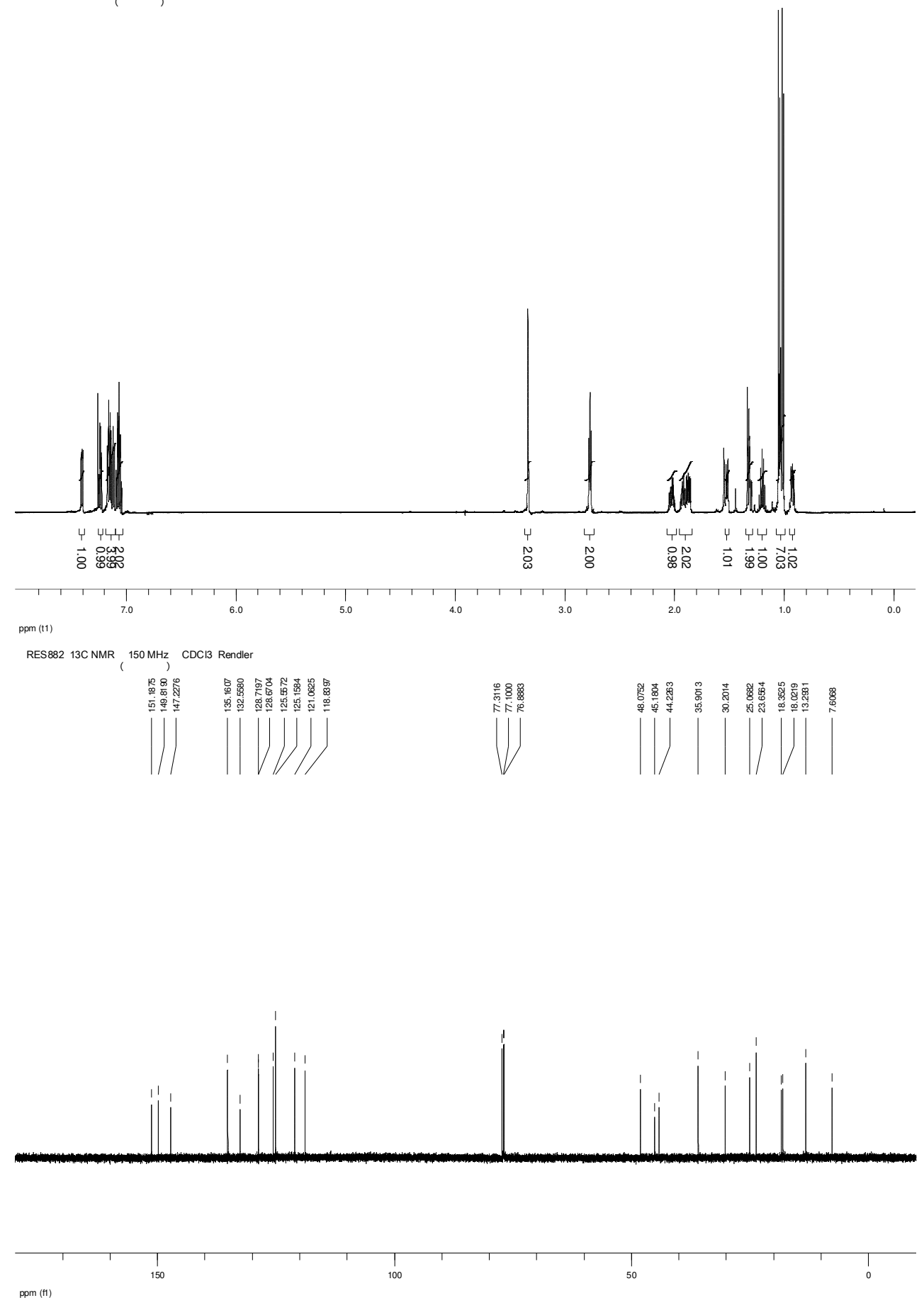

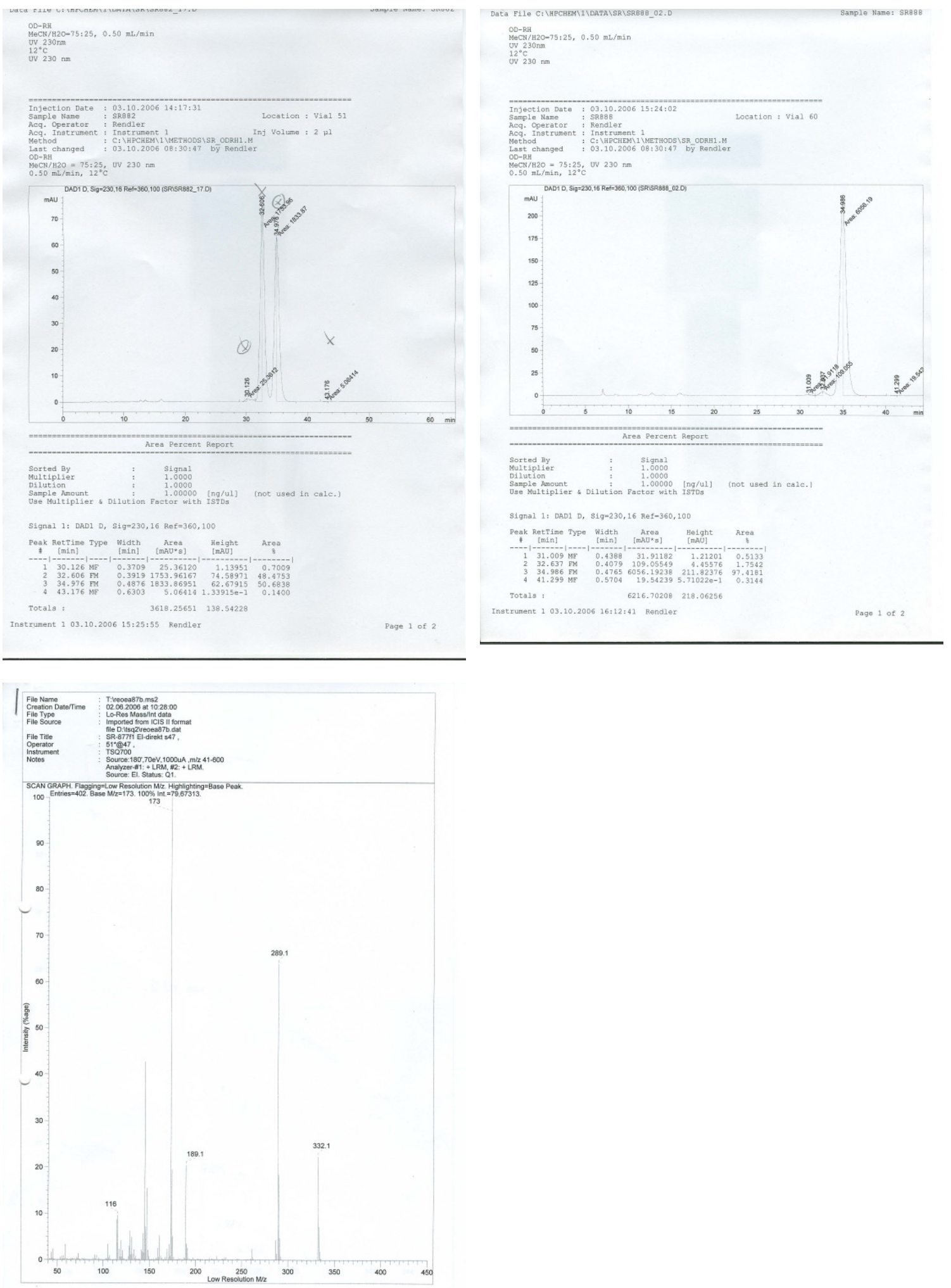


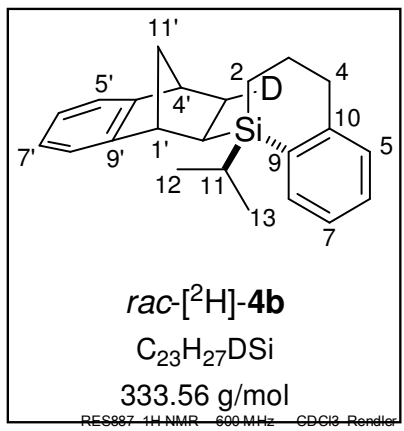

rac- $\left(1 R^{\star}, 2 R^{\star}, 4 S^{\star}, \mathrm{Si} R^{\star}\right)-3$ '-[2H]-1-Isopropyl-1-(1,2,3,4-tetrahydro-1,4methano-naphthalen-2-yl)-1-sila-1,2,3,4-tetrahydronaphthalene (rac$\left.3^{\prime}-[2 \mathrm{H}]-4 b\right)$
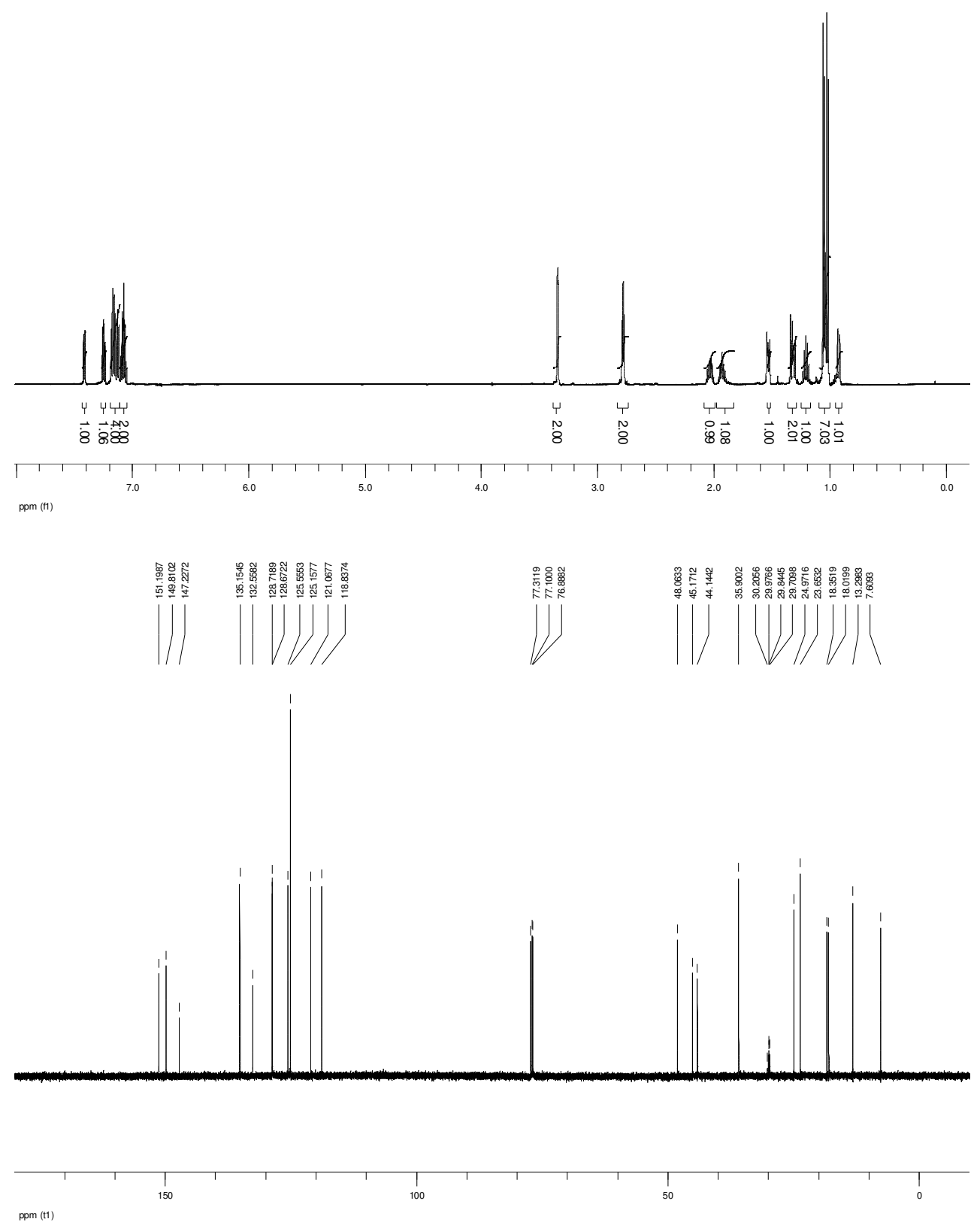

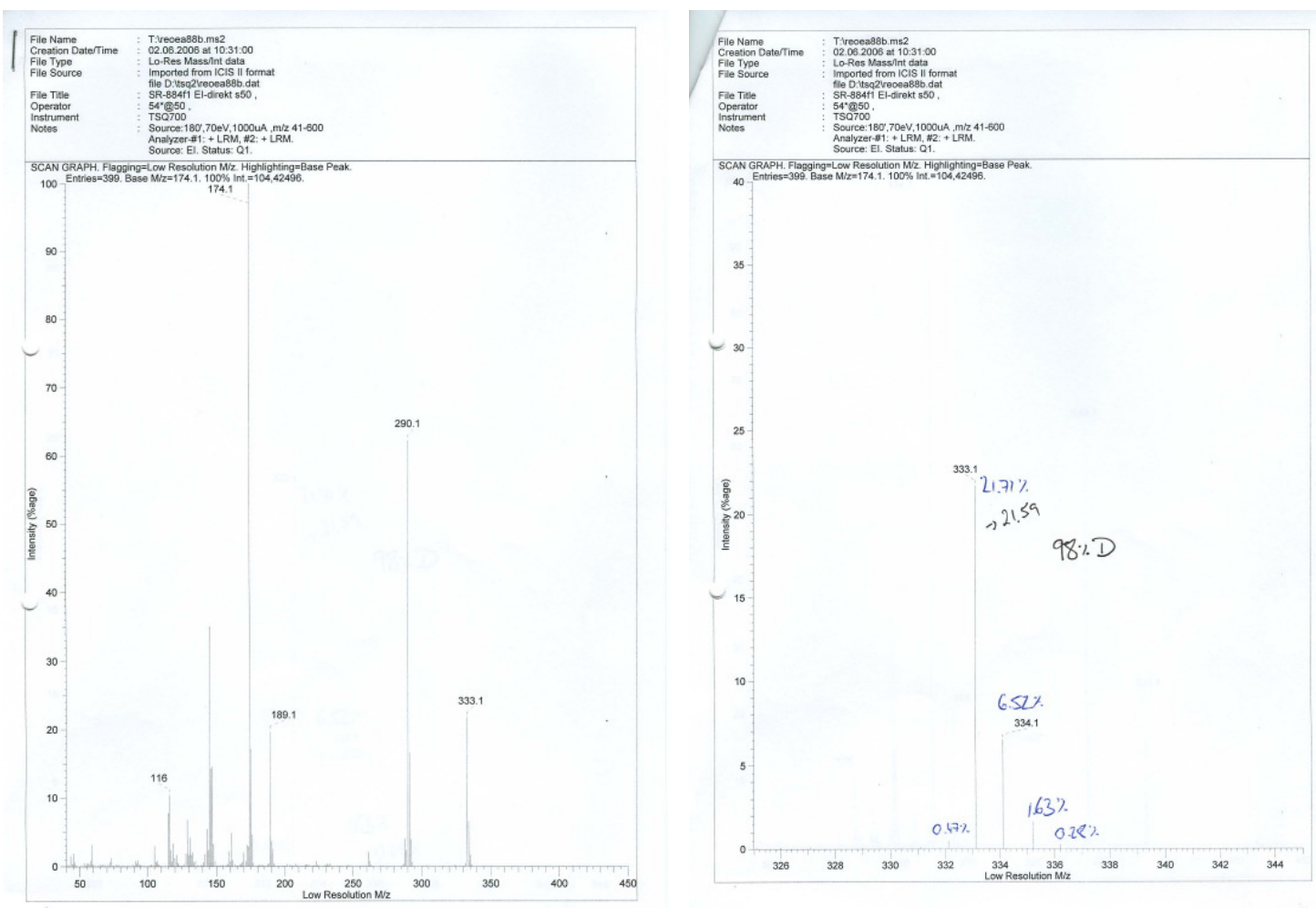


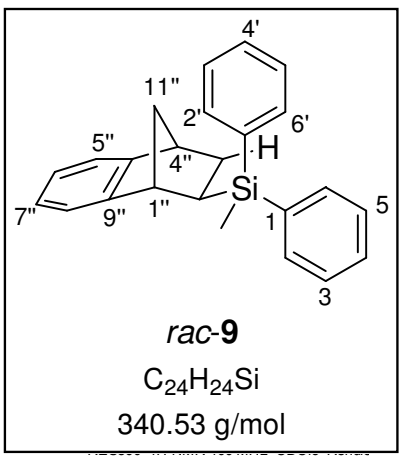

rac-(1S $\left.{ }^{\star}, 2 R^{\star}, 4 S^{\star}\right)-1-M e t h y l-1,1-d i p h e n y l-1-(1,2,3,4-t e t r a h y d r o-1,4-$ methano-naphthalen-2-yl)silane (rac-9)

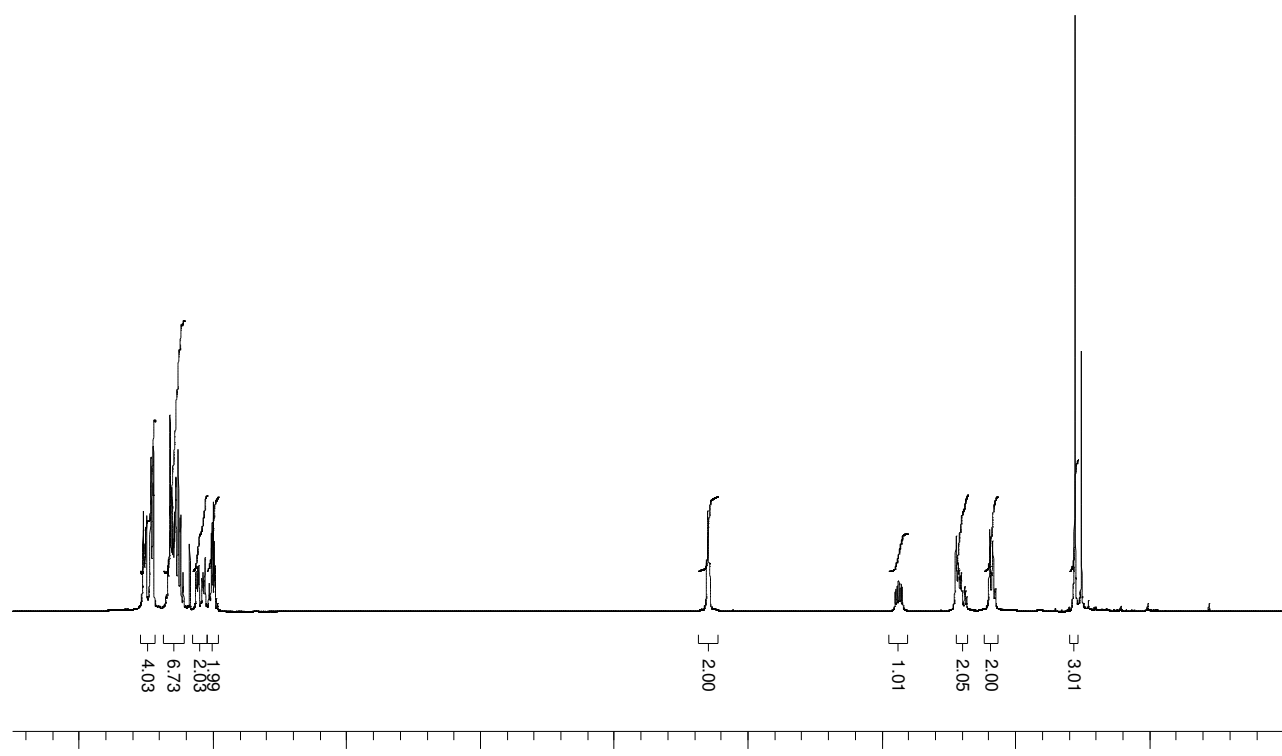

$$
\text { ppm (11) }
$$

RES860 13C NMR $100 \mathrm{MHz}$ CDCl3 Rendler
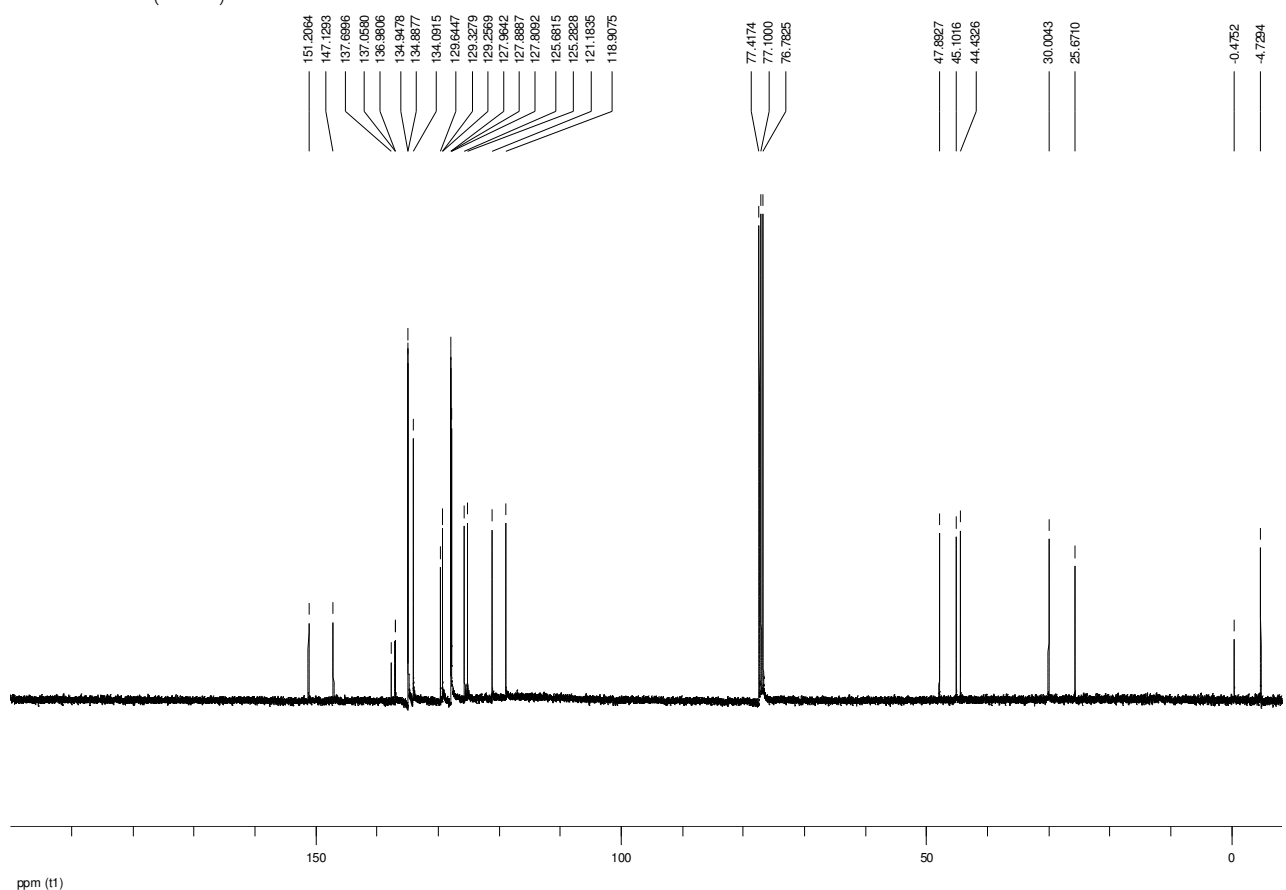


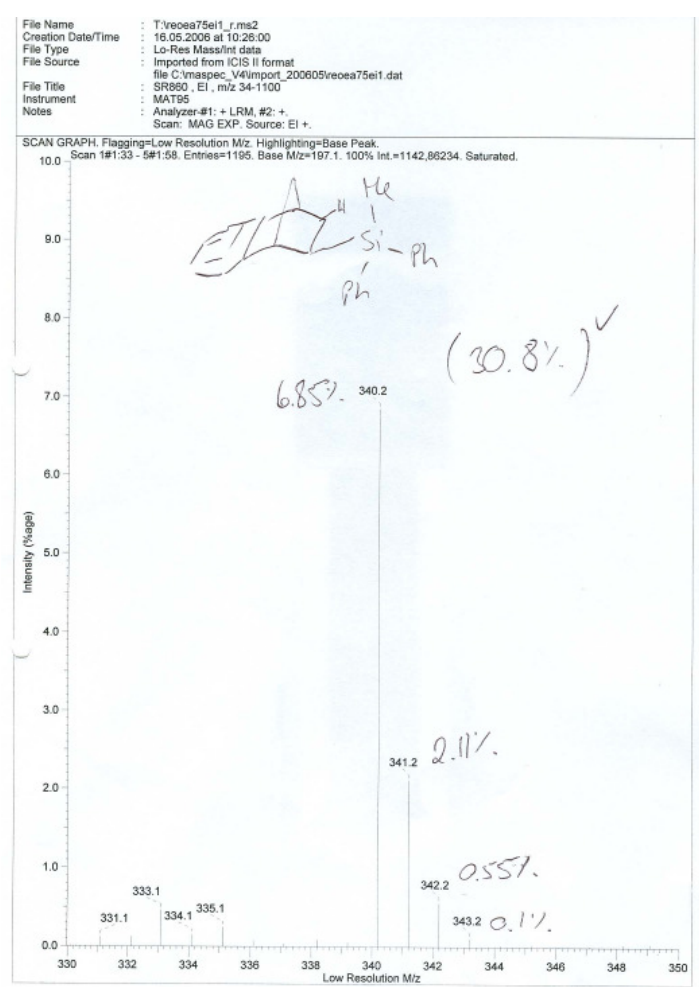




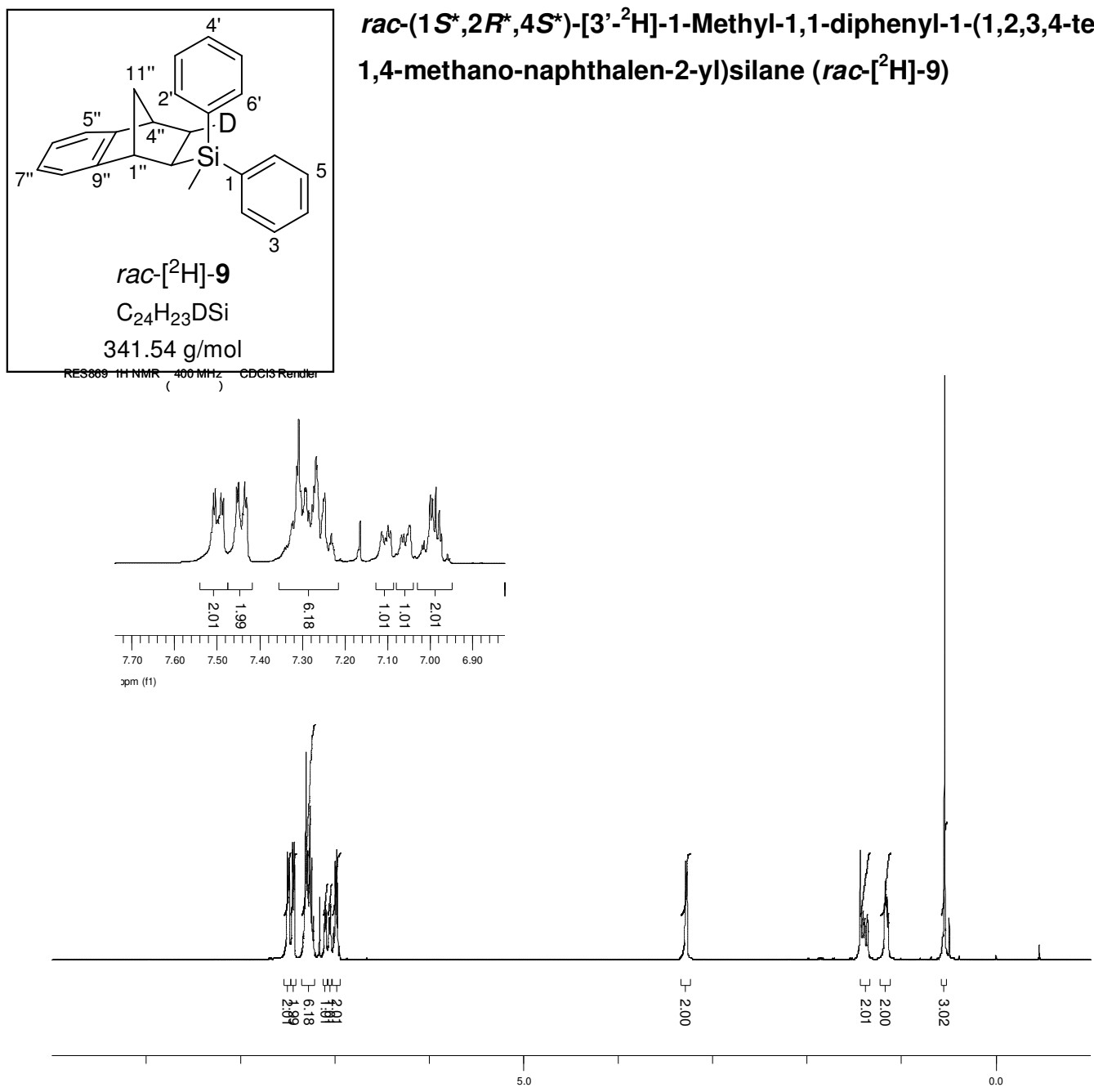

ppm (11)

RES869 $13 \mathrm{C} \mathrm{NMR}, 100 \mathrm{MHz}$ CDC13 Rendler
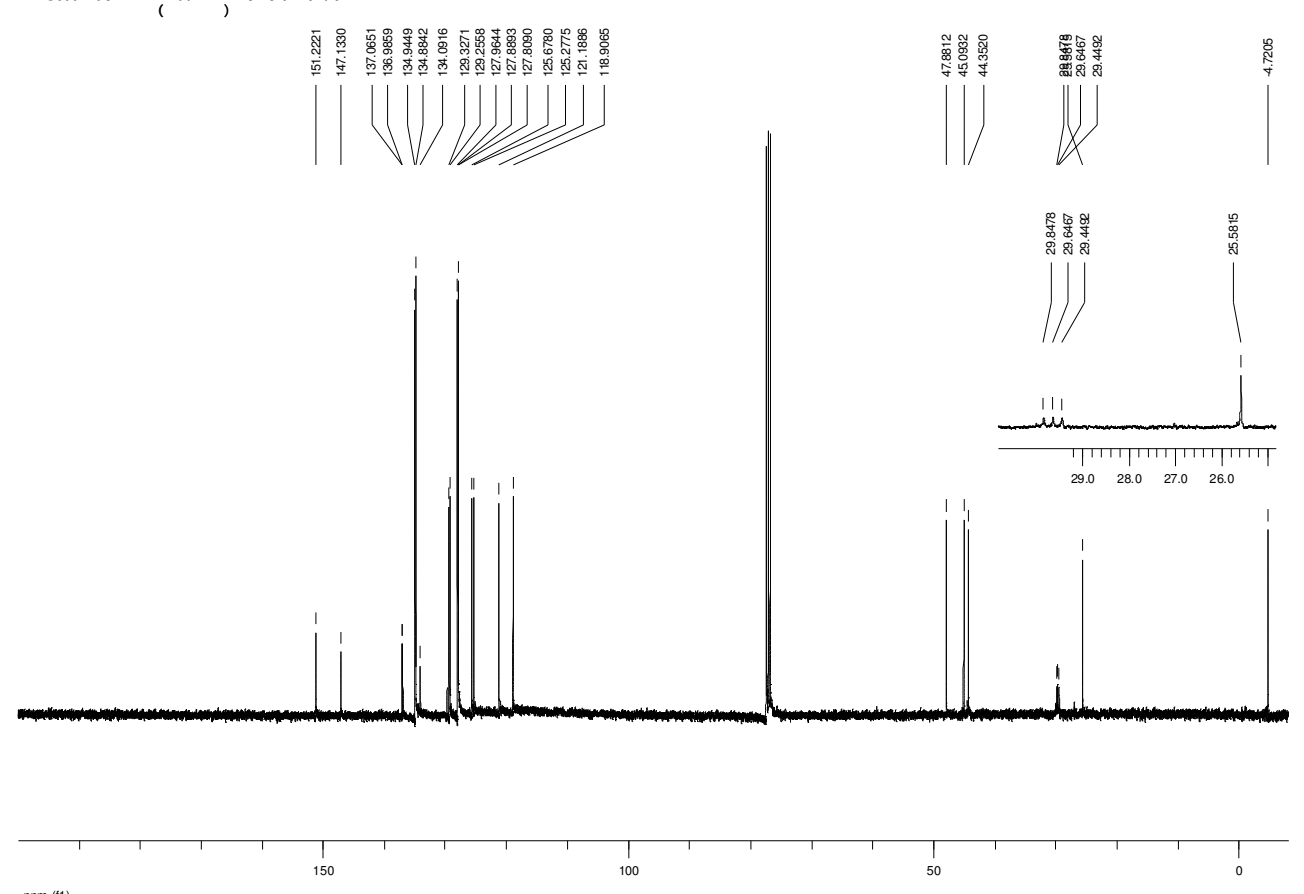

ppm (11) 


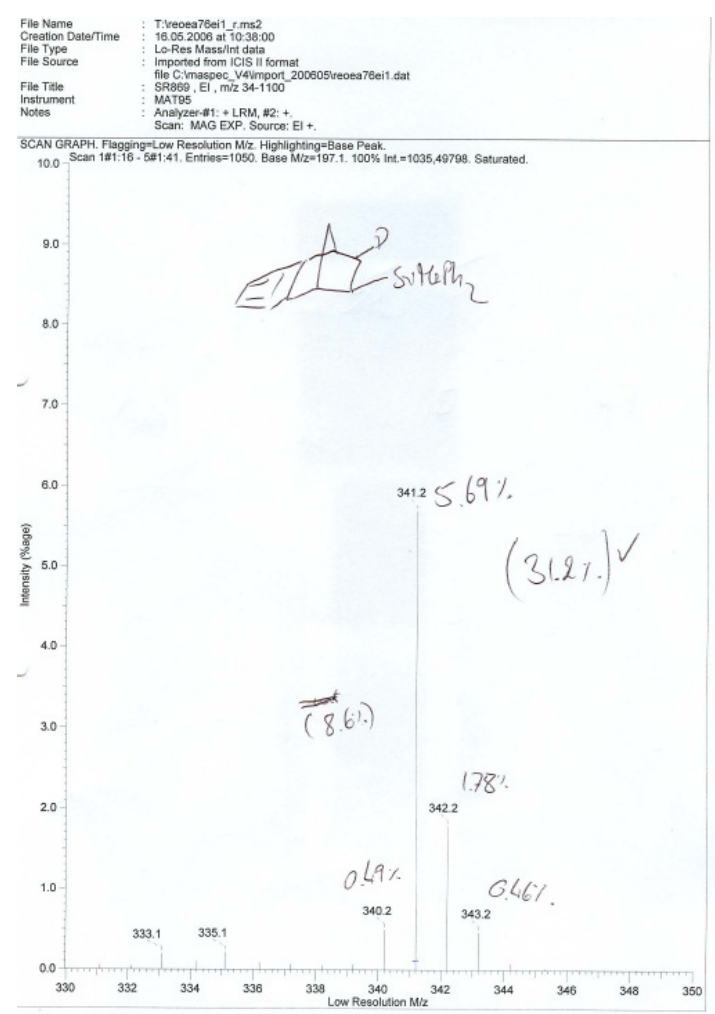

